NBER WORKING PAPER SERIES

\title{
INVESTIGATING THE DYNAMIC EFFECTS OF COUNTERFEITS WITH A RANDOM CHANGEPOINT SIMULTANEOUS EQUATION MODEL
}

\author{
Yi Qian \\ Hui Xie \\ Working Paper 16692 \\ http://www.nber.org/papers/w16692 \\ NATIONAL BUREAU OF ECONOMIC RESEARCH \\ 1050 Massachusetts Avenue \\ Cambridge, MA 02138 \\ January 2011
}

The authors would like to thank the participants at the Marketing Science Conference at Michigan, the seminar participants at Harvard Econometrics, UIUC, and UIC, especially to Gary Chamberlain, Guido Imbens and Xuming He, for helpful comments. We are also grateful to Greg Allenby, Ulf B" ockenholt, Eric Bradlow, Yuxin Chen, and Karsten Hansen for valuable comments. Comments are welcomed and can be sent to yiqian@kellogg.northwestern.edu. The views expressed herein are those of the authors and do not necessarily reflect the views of the National Bureau of Economic Research.

NBER working papers are circulated for discussion and comment purposes. They have not been peerreviewed or been subject to the review by the NBER Board of Directors that accompanies official NBER publications.

(C) 2011 by Yi Qian and Hui Xie. All rights reserved. Short sections of text, not to exceed two paragraphs, may be quoted without explicit permission provided that full credit, including $\odot$ notice, is given to the source. 
Investigating the Dynamic Effects of Counterfeits with a Random Changepoint Simultaneous Equation Model

Yi Qian and Hui Xie

NBER Working Paper No. 16692

January 2011

JEL No. O34

\begin{abstract}
Using a unique panel dataset and a new model, this article investigates the dynamic effects of counterfeit sales on authentic-product price dynamics. We propose a Bayesian random-changepoint simultaneous equation model that simultaneously takes into account three important features in empirical studies: (1) Endogeneity of a market entry, (2) Nonstationarity of the entry effects and (3) Heterogeneity of the firms' response behaviors. Besides accounting for the endogeneity of counterfeiting, the proposed methodology improves the estimation of dynamic effects under heterogeneous response times by firms. We identify both a temporary negative short-term effect and a stable positive long-term effect of counterfeit sales on the authentic prices. Such effect estimates are biased in the OLS model and attenuated in a standard IV model. The findings help to unify two strands of I.O. theories on the pricing effects of competition. Finally, our analysis identifies considerable heterogeneity in authentic firms' response behaviors (both response time and magnitude), and the hierarchical structure of our model enables a study of the drivers of the heterogeneity. This study casts managerial insights on effective brand protection and management strategies that can be tailored to each type of firms. The method illustrated provides a new approach to use field data to study the determinants of a firm's response time, an important dimension of management strategy. In particular, firms with more human capital or less diversification from infringed markets were faster in responding and differentiating from counterfeits. The proposed framework can be widely applied to study dynamic and heterogeneous causal effects of marketing variables.
\end{abstract}

Yi Qian

Department of Marketing

Kellogg School of Management

Northwestern University

2001 Sheridan Road

Evanston, IL 60208

and NBER

yiqian@kellogg.northwestern.edu

Hui Xie

Department of Biostatistics

School of Public Health

University of Illinois at Chicago

huixie@uic.edu 


\section{Introduction}

Brand names have significant economic values and are vulnerable to counterfeiting. The Federal Bureau of Investigation reports that U.S. companies lose $\$ 200-250$ billion annually due to worldwide copyright, trademark, and trade-secret infringements. Significantly, the European Commission (EC) reckons that the value of counterfeiting as a percentage of world trade is growing. Between 1990 and 1999, it doubled from 3.5 percent to 7 percent (Choate 2005). The World Customs Organization (WCO) estimates that over 500 billion Euro of traded world merchandise in 2004 may have been counterfeits (WCO 2004). Most of the counterfeits are charged at extremely low prices, e.g., $\$ 65$ for a counterfeited LV handbag whose original price is $\$ 1200$, or $\$ 5$ for Windows 95 software only after days it was introduced in the U.S. for $\$ 85$ (Choate 2005). The entry of counterfeiters with fractionally low prices can have two opposite impacts on the authentic prices: On the one hand, their entry potentially exerts competitive pressure on authentic prices. Authentic prices could also drop as a result of limit (predatory) pricing strategy (Carlton and Perloff, 2005). On the other hand, their entry may lead to increases in authentic prices due to the segmentation of price-sensitive and insensitive consumers in the market, or due to authentic producers' innovations and self-differentiation mechanics to alleviate competition (Qian 2008). The former competitive effect is likely to take place immediately upon entry, while the latter effect may arrive with some lags. This lag differs from company to company due to inherent firm heterogeneity in their ability to respond to market shakeups caused by counterfeit entry. Therefore, how much and when authentic firms' marketing norms (e.g. prices) change in response to the counterfeit entry are both interesting and pertinent questions to address.

In evaluating the causal effects of counterfeit entry on the authentic price outcome, it is worth noting two important challenges. First, counterfeit entry is unlikely to be exogenous. Counterfeiters are more likely to infringe upon a brand if the authentic product is easier 
to imitate, has a larger markup, or if the brand management is worse. Under such circumstances, counterfeit entry will be correlated with authentic prices and profits. However, a causal link cannot be inferred from this correlation. Qian (2008) adopts the Instrumental Variable (I.V.) identification strategy. Instrumental variables are correlated with the endogenous treatment variable but uncorrelated with the outcome of interest conditional on the treatment variable. One can subsequently use the instrumental variables to tease out the exogenous component of the treatment variation and identify the causal effect. The I.V. method is widely applied in economic and marketing studies.

The second challenge arises because various authentic firms respond to their own counterfeit entry with different time lags. It is natural for the authentic firms to take time to analyze the changing business environment, design corresponding strategies, and implement them in practice. The stable long-term effect follows only with some delay in time, which we refer to as response time. Such delay in response is frequently observed. Robinson (1988) noted a pattern of delayed responses by the incumbent firms based on studying 199 new product entries in a start-up business database. Even defensive price reactions, which are the easiest to implement, are also often delayed. Bowman and Gatignon (1995) also observed delayed response in the PIMS database. In these scenarios, the effect of market change is nonstationary in time in that a study unit (e.g., a firm) changes its state underlying the outcome of interest (e.g., price) at a random time point. Such phenomenon is not restricted to studies on firms, but is also prevalent in studies involving other marketing players, e.g., dynamic effects due to delayed response of consumers to marketing efforts (Kotler 1971). Fader, Hardie and Huang (2004, hereafter referred to as FHH) highlight the importance of taking the nonstationary consumer behavior process into account when forecasting new product sales. In summary, the dynamic effect of a market change is a prevalent phenomenon in practice and is an important subject to study. 
A simple strategy to handle the dynamic effects is to pre-specify a common timepoint where the short-term and long-term effects of the market change can be separated, and then posit an econometric model that enables separate estimation of short-term and long-term effects. This analytic strategy, albeit simple, has the following three limitations.

First, pre-specifying the separating time point may not be easy and requires considerable prior knowledge on the underlying marketing activities. It would be preferable to have a data-driven approach to automatically select the timepoint that separate out the short- and long-term effects of market change.

Second and more importantly, it is often the case that there exists a significant amount of heterogeneity among different units (e.g. firms) in their response times. For example, Bowman and Gatignon (1995) documented a heterogeneous pattern of delayed responses in the widely analyzed PIMS database: the defensive responses to new product entries occurred after more than a year in $28.7 \%$ of the cases, between six months and a year in $18 \%$ of the cases, and immediately in only $13.4 \%$ of the cases. We also observe a pattern of heterogeneous response time in our dataset. It is well known that heterogeneity is common in emprical studies and modeling the heterogeneity can lead to improved prediction. For example, FHH (2004) shows that modeling the changepoints that are heterogeneous among consumers can substantially improve new product sales forecast. Similarly, when there exists such heterogeneity among units in their response time, explicitly modeling the heterogeneous response time can lead to substantially improved estimation of dynamic effects of a market change, and consequently can have more accurate policy implications. For example, we find in our study that ignoring the heterogeneity of response time can attenuate the effect estimate of both short-term and long-term effects. Such attenuation is more serious in evaluating the derterminants of firms' differential response behaviors. We find in our study that the simple strategy to investigate dynamic effects misassesses the effects of several factors on marketing 
responses and is not able to identify their significant effects.

Third, in many cases, how fast a firm responds to a market change is an important dimension of management strategy, and therefore questions such as what determines firms' ability to respond to the market change are of considerable interest (e.g., Bowman and Gatignon 1995). Specifying a common response time ignores this heterogeneity and as a result provides no basis to study such questions. An alternative approach is to employ a distributed lag regression model. However, prior information on response time is rarely available and thus it can be difficult to specify a sensible lag function. Moreover, neither of the above methods is satisfactory if one is interested in studying the individual response times.

Our solution to the above challenge is to explicitly model the unit-specific response time as a changepoint problem. The general class of the changepoint model is well suited for modeling parameter changes in econometric models. There has been extensive research on the changepoint model with early applications in statistics and economics (e.g. Smith 1975, Barry and Hartigan 1993, Carlin et al. 1992, Lange et al. 1992, Bai 1997, Chib 1998). There are important innovations of changepoint modeling in recent marketing literature. Böckenholt and Dillon (1997), Poulsen (1990) and Ramaswamy (1997) use the hidden Markov Models to study the changes in segment memberships over time, which can be viewed as a special case of more general changepoint process models. FHH (2004) develop a dynamic changepoint model that allows the changepoint process itself to evolve over time. They show that the dynamic changepoint model improves the new product sales forecasting. The method has been further studied and extended by Schweidel and Fader (2009). Neelamegham and Chintagunta (2004) and Van Heerde, Mela and Manchanda (2004) extend the Dynamic Linear Model to model new product sales. Fong and DeSarbo (2007) study Bayesian variable selection problems in multiple regression models with changepoints. Our approach is more 
similar to that of FHH (2004) in that we explicitly model the random changepoints which are heterogeneous among the study units. However, unlike FHH (2004) which focuses on forecasting new product sales, the focus of our study is on an accurate ascertainment of dynamic and heterogeneous causal effects of a market change. As such, we must explicitly deal with the endogeneity issue, an important feature in empirical studies. To the best of our knowledge, the endogeneity issue in a changepoint model framework has not been dealt with previously, although its importance has been discussed in FHH (2004).

A key contribution of our study is to provide a tractable methodological framework to achieve consistent and efficient estimation of the dynamic causal effects of an endogenous treatment variable on marketing responses. Specifically, we propose a random-changepoint simultaneous equation model (RC-SEM) to properly measure dynamic effects of an endogenous treatment on the outcome. Our modeling approach jointly models the marketing response, the endogenous treatment variable and the latent unit-specific response time. Therefore the model extends the traditional changepoint model to allow for endogeneity. Another useful feature of our model is that we extend the changepoint model to allow for covariates explaining the inter-firm differences in their response times. Our analysis demonstrates that the proposed model minimizes the bias on the entry effect estimates and can allow us to investigate the determinants of inter-firm difference in their response time. More specifically, we summarize the features and benefits of our modeling framework below.

First, it allows for an automatic and cleaner separation of different stages of the effects of a market change. As explained above, failure to properly account for unit-specific response times can lead to biased estimates of dynamic effects of a market change, thus leading to considerable loss of power for detecting a change in the marketing response. Our approach explicitly models the unit-specific changepoint, thereby providing a much cleaner assessment of dynamic effects. We are able to delineate the short-term pricing responses to counter- 
feiting as well as the stabilized long-term effect, a feature that would have been washed out without appropriately accounting for the heterogeneous response time. This methodological advantage precisely enables us to unify two strands of IO literature and resolve the empirical controversy in the pricing impacts and responses in the face of copycat entries (Pauwels and Srinivasan 2004, Deleersnyder et al 2007 vs. Rao and Monroe 1996, Hoch and Banerji 1993). Furthermore, the changepoint model is a data-driven procedure that automatically detects the changepoint in the outcome. This is particularly useful if there is no strong prior belief on the possible positions of changepoints, and one would like to minimize the input of researchers in this modeling aspect. ${ }^{1}$

Second, it models the endogeneity of the treatment variable. As explained above, the counterfeit entry is likely to be endogenous, and failure to account for the endogeneity can lead to misleading assessment of dynamic causal effects of counterfeit entry. In our analysis we integrate the changepoint model with a simultaneous equation model, thereby simultaneously accounting for both endogeneity and nonstationary effects of counterfeit entry. The need to account for both endogeneity and nonstationarity is not limited to our dataset. For example, as discussed in FHH (2004), both the distribution build and competitive effects could lead to endogeneity issues in new product sales forecasting. Our model provides a framework based on which the endogeneity of a market change is accounted for and therefore cleaner assessment of the dynamic causal effects of market change can be made. Given that both the endogeneity and nonstationary effects of a market change are important and prevalent in empirical studies, the extension would be a useful generalization.

Third, our model enables recovery of individual-specific estimates of how much firms' price respond to entry by counterfeits. We are then able to explore what are the firm characteristics that drive such heterogeneity in pricing responses to counterfeiting. Discovery

$\overline{{ }^{1} \text { Of course, our Bayesian approach allows incorporating such prior belief, if it exists, through proper specification of model }}$ priors. 
of these moderating factors assists in understanding effective brand management strategies that can be tailored to each type of firms. The findings are therefore of keen interests to academics and practitioners and the framework can be applied to understand strategic responses under other contexts, such as pricing of national brands upon entry of imitative store brands.

Fourth, the modeling framework allows us to study how fast firms respond to market change, an important dimension of a reactive strategy, and to study the determinants of response speed. Given the staggering patterns of defensive responses by incumbents as identified in prior literature (Robinson, 1988; Bowman and Gatignon, 1995; Qian, 2008), the following questions naturally arise: Why do some incumbents choose to respond immediately to the competitive entry while others delay their responses? The response time likely represents the heterogeneity of the units, such as firms, in their ability to adapt to the constantly changing environment. Thus, the response time can be of great interest to identify institutional features that prompt effective and swift business strategy. The importance of response time as a key strategic issue in marketing has been emphasized in previous literature (Robinson 1988, Smith et al. 1989, Heil and Robinson 1991, Bowman and Gatignon 1995). As pointed out by the authors, the study of similar issues such as the competitor's response in other context can be very useful to design informed marketing strategies. The previous studies typically use survey data to investigate the questions. For example, Bowman and Gatignon (1995) use survey data to study the determinants of competitor response time to a new product introduction. The survey contains a question that asks a firm how long each of a firm's leading competitors took to respond in a visible manner when the firm introduced new products. As noted by the authors, one methodological limitation of this approach relates to various potential response biases in the dependent variable? Using our

\footnotetext{
${ }^{2}$ For example, the authors discusses that the interview question is on the firm's customers but the respondent is the firm introducing the new product. A threat to content validity is that the respondent does not have perfect complete knowledge of
} 
proposed model, researchers have an alternative method that uses the field data to study the similar question. Specifically the method uses the changepoint modeling technique to infer latent response times from the trajectory of the marketing response observed over time. The hierarchical modeling structure allows us to further study the determinants of firms' response times. We hope that the proposed method expands the set of tools that can be used by the researchers to study similar questions.

Another contribution of the study is the scope of the dataset, which is a large national sample that includes 31 branded shoe manufacturing firms and their counterfeiters in China. Both multinational brands in China and Chinese-originated brands are sampled through the stratified random sampling method (Qian 2008). Twenty-two out of the total 23 large branded firms in China are captured, together with a random sample of smaller ones. Detailed financial statements of each sampled company and their counterfeiters are obtained from a 12-year window from 1993-2004. This is a unique dataset that overcomes severe data limitations common in the underground economics. Our analysis here differs from Qian (2008) in several important ways. First, we tackle a different research question. Qian (2008) explores the overall impacts of entry by counterfeiters on a portfolio of marketing outcomes. The study provides comprehensive first-cut analyses on the stabilized authentic quality, price, sales, self-enforcement expenditure, and profits years after being infringed. It opens doors to more detailed analyses like the current study, where we focus on the important pricing strategies against counterfeits. We carefully analyze the longitudinal pricing responses by the authentic brands and explicitly model the heterogeneous response times between firms. The new methodology enables us to distinctively identify the negative short-term effect and positive long-term effect of counterfeits on authentic prices. The empirical findings unify two strands of theoretical predictions in the industrial organization and marketing litera- 
ture. Second, the explicit modeling of the latent response time also allows us to study how fast firms respond to the entry of counterfeits, an important marketing strategy not yet studied in Qian (2008). Third, using the hierarchical Bayesian method, we investigate the heterogeneous effects of counterfeit entry on both the price outcome and firms' response time. This provides additional insights on the firm behavior when faced with the threat of counterfeiting. This also sheds lights on the determinants of interfirm differences of their response magnitudes and speed. Last but not least, we propose a new methodology to study latent response times using field data and to delineate dynamic effects of an endogenous marketing variables.

The rest of the paper is structured as follows: Section 2 describes the proposed model: RC-SEM. Section 3 describes the model inference with more details given in Appendix. Section 4 applies the RC-SEM to the Chinese Shoe Market Data. In Section 5 we conduct a simulation study to evaluate the performance of the method. We then conclude with a discussion in Section 6.

\section{Modeling Approach}

In this section we develop the proposed methodology to investigate the dynamic effects of counterfeit entry using a dataset of the Chinese shoe industry. The panel data consist of annual average prices, costs and sales for 31 authentic branded companies and their counterfeiters from the year 1993 to 2004. Qian (2008) includes more details of the dataset. In this article, we focus on studying the dynamic and heterogeneous causal effects of counterfeit entry and sales on the authentic product prices using the proposed RC-SEM framework. As aforementioned, one question of great interest is to investigate the effects of counterfeit entry on authentic firms' pricing strategy. As a preliminary analysis, Figure 1 presents the time plot of the average log deflated authentic high-end product prices. Specifically the figure 
plots the regression coefficients on a set of dummies indicating the number of years relative to the year of counterfeit entry with the log deflated authentic price as the response variable. The plot suggests the presence of dynamic effects of counterfeit entry on authentic prices: there was a reduction in the average authentic prices within first two years of the counterfeit entry, after which there was an increase in the average authentic prices.

The above simple analysis, though informative and useful, has some important limitations. Notably, the analysis has not yet accounted for the potential endogeneity issue of counterfeit entry. Secondly, although the analysis reveals a potential changepoint in the average price profile, it ignores the heterogeneity of the changepoint among the firms, and assumes that all the authentic firms took the same amount of time to respond to their counterfeit entries. As aforementioned and further demonstrated in the following sections, ignoring the heterogeneity can attenuate the dynamic-effect estimates of counterfeit entry. Bias also arises when assessing the effects of various factors on the marketing responses. Furthermore, such preliminary analysis does not allow us to study what affects a firm's response time to counterfeit entry.

In order to overcome the limitations of the preliminary analysis, we propose a randomchangepoint simultaneous equations model to investigate the dynamic effects of counterfeit entry. Our modeling framework jointly models the panel price profiles of the authentic firms evolved over time, the quantity of counterfeits faced by the authentic firms as well as the latent random changepoints in the panel price profiles caused by the heterogeneous response times of the authentic firms to the counterfeits products. The model accounts for several important features in the data: endogeneity, heterogeneity as well as the nonstationary parameters due to changepoint. We describe the overall model in the following two subsections. 


\subsection{Within-firm Model}

Let $Y_{1}, \ldots, Y_{N}$ denote the outcome vectors on a random sample of $N$ units (i.e. firms in our application), where $Y_{i}=\left(Y_{i 1}, \ldots, Y_{i J}\right)$ is a $J$-dimensional panel outcome vector for the $i$ th unit, $i=1, \ldots, N$ and $N=31, J=12$ in our application. ${ }^{3}$ We assume that the vector outcome $Y_{i}$ is generated from the following structural model with changepoint:

$$
Y_{i j}= \begin{cases}X_{i j}^{T} \beta_{1 i}+U_{i j}^{T} \alpha_{i}+W_{i j}^{T} \gamma+\epsilon_{i j}^{Y} & j=1, \ldots, T_{i}-1 \\ X_{i j}^{T} \beta_{2 i}+U_{i j}^{T} \alpha_{i}+W_{i j}^{T} \gamma+\epsilon_{i j}^{Y} & j=T_{i}, \ldots, J\end{cases}
$$

where the covariate $X$ includes variables that are believed to have potentially differential short-term $\left(\beta_{1 i}\right)$ and long-term $\left(\beta_{2 i}\right)$ impacts on the outcome. In our application, $X$ refers to the quantity of counterfeit products in the market faced by the authentic firm $i$, divided by the sale quantity of this authentic firm. It is clear from the model specification that the commonly used static model is obtained by setting $\beta_{1 i}=\beta_{2 i}$. Therefore, our model nests the commonly used static model as a special case. As discussed in the Introduction section, we expect $X$ to have dynamic effects on the outcome (i.e., $\beta_{1 i} \neq \beta_{2 i}$ ). When $\beta_{1 i}$ is different from $\beta_{2 i}$, the static model can give misleading estimates about the effects of $X$ on the outcome $Y$. For example, when $\beta_{1 i}$ and $\beta_{2 i}$ are of opposite sign, it is likely that the static model that assumes $\beta_{1 i}=\beta_{2 i}$ would greatly attenuate the effect estimate. Therefore, our model development will be based on the more general model that allows for the possibility of dynamic effects. The other variables in the model, $U$ and $W$, include those observed exogenous variables. $U$ includes those variables whose effects on $Y$ are heterogeneous among the firms. This may include the unit dummy variables to capture the time-constant heterogeneity across units that are unobserved to the researchers, e.g., the attributes of individual-firm management, etc. $W$ may include the time dummy variables to capture the common market shocks to all the units at a given time.

\footnotetext{
3 Although our dataset is a balanced data in which each firm has the same number of years of observations, the proposed method is general and can be applied to unbalanced data as well.
} 
In our modeling framework, we allow $X$ to be endogenous. We follow the approach of Amemiya (1985) and introduce a latent variable $X_{i j}^{*}$ as follows

$$
\begin{aligned}
& X_{i j}= \begin{cases}X_{i j}^{*} & \text { if } X_{i j}^{*} \geq 0 \\
0 & \text { if } X_{i j}^{*}<0\end{cases} \\
& X_{i j}^{*}=\delta Z_{i j}+\epsilon_{i j}^{X},
\end{aligned}
$$

where $X_{i j}^{*}$ is a latent variable that determines the observed variable $X_{i j}$ according to Equation (2), and $Z_{i j}$ is a vector of exogenous instrumental variables that relate to $X_{i j}^{*}$. The endogeneity of $X_{i j}$ is modeled by the correlation between the error terms $\epsilon_{i j}^{X}$ and $\epsilon_{i j}^{Y}$, which are assumed to follow a bivariate normal:

$$
\left[\epsilon_{i j}^{Y}, \epsilon_{i j}^{X}\right] \sim N\left(0, \Sigma_{\epsilon}\right), \quad \Sigma_{\epsilon}=\left(\begin{array}{cc}
\sigma_{11} & \sigma_{12} \\
\sigma_{12} & \sigma_{22}
\end{array}\right)
$$

In this model, $X$ is endogenous when $\sigma_{12}$ is nonzero.

In the above simultaneous equation model, we are primarily interested in measuring the causal effects of changing $X$ on the outcome $Y$ using a panel data where at least some of the units in the sample experienced the change of $X$ value over the period under examination. The model allows dynamic effects of changing $X$ on $Y$ : a short-term effect denoted by the parameter $\beta_{1 i}$ and a long-term effect denoted by the parameter $\beta_{2 i}$. The model assumes that the latent time point at which two effects are separated for the $i$ th firm is $T_{i} \geq T_{i 0}$, where $T_{i 0}$ is the time of counterfeit entry for the $i$ th firm. Let $\tau_{i}=T_{i}-T_{i 0}$, and then $\tau_{i}$ denotes the time for the $i$ th firm to respond: the time from the counterfeit entry to the earliest time point when the long-term effect is manifested in the outcome variable. We note here the one-to-one correspondence between $\tau_{i}$ and $T_{i}$. Furthermore, both $\tau_{i}$ and $T_{i}$ are unknown and will be inferred from the data using the changepoint modeling technique. It is often the case that the long-term effect is very different from the short-term effect. Figure 2 represents two firms with different response times, $\tau_{1}$ and $\tau_{2}$. An ad-hoc method that 
specifies a common response time for both firms, say $\tau_{c}$, ignores this heterogeneity and can lead to biased estimates of the dynamic effects of $X$ on $Y$, as will be shown later. Our model aims to separate out these two effects more cleanly by explicitly modeling the underlying unit-specific changepoint. An added benefit of doing this is to provide estimates of response times and to be able to study what affects firms' response times.

To model the response time as a function of firm-characteristics, we assume that there is a continuous response time variable, $\tau_{i}^{*}$, for the firm $i$. Because the observed value is determined by the coarsened units of the outcome, e.g. years in our dataset, the response time $\tau_{i}^{*}$ is observed to fall in a certain interval with its actual value unobserved. Such data often occurred in other studies, such as the length of time with the present employer or duration of unemployment in survey studies. In our case, the grouped value $\tau$ is determined by its underlying value $\tau^{*}$ according to the following set of rules:

$$
\tau_{i}= \begin{cases}0 & \text { if } \tau_{i}^{*} \leq 0 \\ k & \text { if } k-1<\tau_{i}^{*} \leq k, \quad 1<k \leq J-T_{i 0} \\ J-T_{i 0}+1 & \text { if } \tau_{i}^{*}>J-T_{i 0} .\end{cases}
$$

In the above model, $\tau_{i}=0$ implies that there is no short-term effect and long-term effect kicks in immediately for this firm; $\tau_{i}=J-T_{i 0}+1$ implies that a long-term effect has not arrived and we only observed a short-term effect over the study period. Both of these two cases are unlikely in our dataset since (1) it usually takes time for a firm to design responding strategy and (2) our panel spans twelve years and all firms, if infringed, have at least five years of observations after counterfeit entry, which is long enough for firms to respond. However, for model completeness, we include these two cases in our model development.

We derive the likelihood for the above unit-level model as follows.

$$
\begin{aligned}
f\left(Y_{i}, X_{i} \mid \tau_{i}^{*}, \beta_{1 i}, \beta_{2 i}, \alpha_{i}, \gamma, \delta, \Sigma_{\epsilon}\right) & =\int \sum_{\tau_{i}} f\left(Y_{i}, X_{i}, X_{i}^{*}, \tau_{i} \mid \tau_{i}^{*}, \beta_{1 i}, \beta_{2 i}, \alpha_{i}, \gamma, \delta, \Sigma_{\epsilon}\right) d X_{i}^{*} \\
& =\int \sum_{\tau_{i}} f\left(Y_{i}, X_{i}, X_{i}^{*} \mid \tau_{i}, \beta_{1 i}, \beta_{2 i}, \alpha_{i}, \gamma, \delta, \Sigma_{\epsilon}\right) f\left(\tau_{i} \mid \tau_{i}^{*}\right) d X_{i}^{*}
\end{aligned}
$$


where the density function $f\left(\tau_{i} \mid \tau_{i}^{*}\right)$ is

$$
f\left(\tau_{i} \mid \tau_{i}^{*}\right) \propto \begin{cases}I\left(\tau_{i}=0\right) & \text { if } \tau_{i}^{*} \leq 0 \\ I\left(\tau_{i}=k\right) & \text { if } k-1<\tau_{i}^{*} \leq k, 1<k \leq J-T_{i 0} \\ I\left(\tau=J+1-T_{i 0}\right) & \text { if } \tau^{*}>J-T_{i 0} .\end{cases}
$$

The conditional density function $f\left(Y_{i}, X_{i}, X_{i}^{*} \mid \tau_{i}, \beta_{1 i}, \beta_{2 i}, \alpha_{i}, \gamma, \delta\right)$ is derived as follows:

(1) $\tau_{i}=0$

This corresponds to the case of immediate response from firm $i$. In our derivation, we write the joint distribution of $f\left(\epsilon_{i j}^{Y}, \epsilon_{i j}^{X}\right)=f\left(\epsilon_{i j}^{X}\right) f\left(\epsilon_{i j}^{Y} \mid \epsilon_{i j}^{X}\right)$ as in Rossi et al. (2005). In this decomposition, $\epsilon_{i j}^{Y} \mid \epsilon_{i j}^{X} \sim N\left(\frac{\sigma_{12}}{\sigma_{22}} \epsilon_{i j}^{X}, \sigma_{1 \mid 2}^{2}\right)$, where $\sigma_{1 \mid 2}^{2}=\sigma_{11}-\frac{\sigma_{12}^{2}}{\sigma_{22}}$. We then have the density function as follows:

$$
\begin{aligned}
f\left(Y_{i}, X_{i}, X_{i}^{*} \mid \tau_{i}=0\right)= & \prod_{j}\left[\left(I\left(X_{i j}=0, X_{i j}^{*}<0\right)+I\left(X_{i j}>0, X_{i j}^{*}=X_{i j}\right)\right)\right. \\
& \left.\phi\left(Y_{i j}-X_{i j} \beta_{2 i}-U_{i j}^{T} \alpha_{i}-W_{i j}^{T} \gamma-\frac{\sigma_{12}}{\sigma_{22}} \epsilon_{i j}^{X} \mid 0, \sigma_{1 \mid 2}^{2}\right) \phi\left(\epsilon_{i j}^{X}=X_{i j}^{*}-\delta Z_{i j} \mid 0, \sigma_{22}\right)\right],
\end{aligned}
$$

where $\phi\left(\cdot \mid \mu, \sigma^{2}\right)$ stands for the density function for normal distribution with mean $\mu$ and variance $\sigma^{2}$.

(2) $0<\tau_{i}<J-T_{i 0}+1$

In this case, we have the density function as follows:

$$
\begin{aligned}
f\left(Y_{i}, X_{i}, X_{i}^{*} \mid \tau_{i}\right)= & \prod_{j<T_{i 0}+\tau_{i}}\left[\left(I\left(X_{i j}=0, X_{i j}^{*}<0\right)+I\left(X_{i j}>0, X_{i j}^{*}=X_{i j}\right)\right)\right. \\
& \left.\phi\left(Y_{i j}-X_{i j} \beta_{1 i}-U_{i j}^{T} \alpha_{i}-W_{i j}^{T} \gamma-\frac{\sigma_{12}}{\sigma_{22}} \epsilon_{i j}^{X} \mid 0, \sigma_{1 \mid 2}^{2}\right) \phi\left(\epsilon_{i j}^{X}=X_{i j}^{*}-\delta Z_{i j} \mid 0, \sigma_{22}\right)\right] \\
& \times \prod_{j \geq T_{i 0}+\tau_{i}}\left[\left(I\left(X_{i j}=0, X_{i j}^{*}<0\right)+I\left(X_{i j}>0, X_{i j}^{*}=X_{i j}\right)\right)\right. \\
& \left.\phi\left(Y_{i j}-X_{i j} \beta_{2 i}-U_{i j}^{T} \alpha_{i}-W_{i j}^{T} \gamma-\frac{\sigma_{12}}{\sigma_{22}} \epsilon_{i j}^{X} \mid 0, \sigma_{1 \mid 2}^{2}\right) \phi\left(\epsilon_{i j}^{X}=X_{i j}^{*}-\delta Z_{i j} \mid 0, \sigma_{22}\right)\right] .
\end{aligned}
$$


(3) $\tau_{i}=J-T_{i 0}+1$

In this case, we have the density function as follows:

$$
\begin{aligned}
f\left(Y_{i}, X_{i}, X_{i}^{*} \mid \tau_{i}\right)= & \prod_{j}\left[\left(I\left(X_{i j}=0, X_{i j}^{*}<0\right)+I\left(X_{i j}>0, X_{i j}^{*}=X_{i j}\right)\right)\right. \\
& \left.\phi\left(Y_{i j}-X_{i j} \beta_{1 i}-U_{i j}^{T} \alpha_{i}-W_{i j}^{T} \gamma-\frac{\sigma_{12}}{\sigma_{22}} \epsilon_{i j}^{X} \mid 0, \sigma_{1 \mid 2}^{2}\right) \phi\left(\epsilon_{i j}^{X}=X_{i j}^{*}-\delta Z_{i j} \mid 0, \sigma_{22}\right)\right] .
\end{aligned}
$$

Therefore, given each possible value of $\tau_{i}$, we can construct the above likelihood. These unit-level likelihood will be combined with the hierarchical prior distribution of unit-level latent data to draw inference on the likely position of changepoints.

\subsection{Between-firm Model}

In this subsection, we consider modeling the firm-level parameters and latent variables, $\left(\alpha_{i}, \beta_{1 i}, \beta_{2 i}, \tau_{i}^{*}\right)$, as a function of firm-level characteristics. The purpose is to study the determinants of the interfirm difference on the short-term effect, long-term effect, and the firms' response times to counterfeit entry. We use the following multivariate normal to model the heterogeneity of these latent variables.

$$
\left[\begin{array}{c}
\alpha_{i} \\
\beta_{1 i} \\
\beta_{2 i} \\
\tau_{i}^{*}
\end{array}\right]=\Pi Z_{i}+\left[\begin{array}{c}
e_{\alpha} \\
e_{\beta_{1}} \\
e_{\beta_{2}} \\
e_{\tau}
\end{array}\right]
$$

where $\Pi$ is a $n_{r} \times n_{z}$ matrix containing the hyperparameters governing the population distribution of firm-level latent variables, $n_{r}$ is the number of these latent variables, $n_{z}$ is the number of variables for firm characteristics (plus an intercept term), and $e=\left(e_{\alpha}, e_{\beta_{1}}, e_{\beta_{2}}, e_{\tau}\right)^{T}$ are random residuals that are assumed to be jointly multivariate normal as

$$
\left(e_{\alpha}, e_{\beta_{1}}, e_{\beta_{2}}, e_{\tau}\right) \sim \operatorname{MVN}\left[(0,0,0,0), \Sigma_{e}\right]
$$


There are two benefits of the between-firm model. One is that this provides a framework for us to investigate the determinants of firms' heterogeneous response behaviors in terms of both their response magnitudes and response speed. Second, this allows one to leverage strength from different firms in the estimation of firm-level models. In this approach, the latent response time $\tau_{i}^{*}$ is treated the same as the other firm-level latent characteristics, $\left(\alpha_{i}, \beta_{1 i}, \beta_{2 i}\right)$. Therefore the model extends the traditional hierarchical model to incorporate the latent response time as an additional dimension of the outcomes to study. These latent variables are inferred from the observed data and the hierarchical model provide a framework to study the determinants of the interfirm differences. In particular, the approach allows one to use field data to study what might affect firms' response times.

The above Equations (1),(2), (3), (4), (5), (8) and (9) specify a probability model describing the data-generating process. The entire parameter vector is $\left(\Pi, \gamma, \delta, \Sigma_{\epsilon}, \Sigma_{e}\right)$. The likelihood for these parameters is as follows:

$$
\begin{aligned}
& L\left(\Pi, \gamma, \delta, \Sigma_{\epsilon}, \Sigma_{e} ; X, Y\right) \\
& \propto \prod_{i} \int f\left(Y_{i}, X_{i} \mid \tau_{i}^{*}, \beta_{1 i}, \beta_{2 i}, \alpha_{i}, \gamma, \delta, \Sigma_{\epsilon}\right) f\left(\alpha_{i}, \beta_{1 i}, \beta_{2 i}, \tau_{i}^{*} \mid \Pi, \Sigma_{e}\right) d \alpha_{i} d \beta_{1 i} d \beta_{2 i} d \tau_{i}^{*},
\end{aligned}
$$

where $i=1, \ldots, N, f\left(Y_{i}, X_{i} \mid \tau_{i}^{*}, \beta_{1 i}, \beta_{2 i}, \alpha_{i}, \gamma, \delta, \Sigma_{\epsilon}\right)$ is specified in Equation (6) and $f\left(\alpha_{i}, \beta_{1 i}, \beta_{2 i}, \tau_{i}^{*} \mid \Pi, \Sigma_{e}\right)$ is the density function of Equation (8) and (9).

\section{Inference}

As shown above, the likelihood for the RC-SEM involves integration and summation over the latent variables $X_{i j}^{*}, \alpha_{i}, \beta_{1 i}, \beta_{2 i}, \tau_{i}^{*}$ and $\tau_{i}$ respectively, which renders inference based on the direct Maximum Likelihood Estimation or Least Square method difficult or even intractable. We use the Bayesian approach for inference. Specifically we use data augmentation technique 
for the estimation of the model (Tanner and Wong 1987). We augment the parameter vector by the latent data described above, and then sample from the joint posterior distribution of model parameters and latent variables. Such a method does not require numerical evaluation of integrals. Moreover, it is straightforward to make inferences on both the population parameters and latent variables under the Bayesian framework. For example, their estimates and the corresponding standard errors can be readily obtained from the posterior draws.

To complete our model, we need to specify the priors for the parameters in the model. Let $\Theta=\operatorname{vec}\left(\Pi^{\prime}\right)$. We assign priors for the model parameters as follows:

$$
\begin{aligned}
& \Theta \sim N\left(\mu_{\Pi}, \Lambda_{\Pi}^{-1}\right), \quad \gamma \sim N\left(\mu_{\gamma}, A_{\gamma}^{-2}\right), \quad \delta \sim N\left(\mu_{\delta}, A_{\delta}^{-1}\right) . \\
& \Sigma_{\epsilon} \sim I W\left(\nu_{\epsilon}, S_{\epsilon}\right), \quad \Sigma_{e} \sim I W\left(\nu_{e}, S_{e}\right),
\end{aligned}
$$

where $I W(\nu, S)$ stands for an inverse-Wishart distribution with $\nu$ degrees of freedom and the scale matrix $S$. The above distributional forms are chosen for priors because these are conjugate priors for deriving the conditionals in our Gibbs sampler described later. In our analysis, the constants in the priors are chosen in a way so that these priors are relatively diffuse. The assignment of values for the constants in these priors is described in Appendix. With the above specified priors, model specification, and observed data $X$ and $Y$, the posterior distribution of the parameters and latent data is as follows, up to a constant:

$$
\begin{aligned}
& \pi\left(\Pi, \gamma, \delta, \Sigma_{\epsilon}, \Sigma_{e}, X^{*}, \alpha_{i}, \beta_{1 i}, \beta_{2 i}, \tau, \tau^{*} \mid Y, X\right) \propto \\
& \prod_{i=1}^{N} f\left(Y_{i}, X_{i}, X_{i}^{*} \mid \tau_{i}, \alpha_{i}, \beta_{1 i}, \beta_{2 i}, \gamma, \delta, \Sigma_{\epsilon}\right) f\left(\tau_{i} \mid \tau_{i}^{*}\right) f\left(\tau_{i}^{*}, \alpha_{i}, \beta_{1 i}, \beta_{2 i} \mid \Pi, \Sigma_{e}\right) \\
& \cdot \pi\left(\Pi \mid \mu_{\Pi}, \Lambda_{\Pi}^{-1}\right) \pi\left(\gamma \mid \mu_{\gamma}, A_{\gamma}\right) \pi\left(\delta \mid \mu_{\delta}, A_{\delta}\right) \pi\left(\Sigma_{\epsilon} \mid \nu_{\epsilon}, S_{\epsilon}\right) \pi\left(\Sigma_{e} \mid \nu_{e}, S_{e}\right) .
\end{aligned}
$$

Although the analytical expression of the posterior distribution is unavailable, we can obtain draws from the posterior distribution of the parameters and the latent data through simulation method. Detailed Gibbs sampler algorithm for model estimation is given in the 
Appendix.

\section{Empirical Analysis}

\subsection{A Bayesian Analysis using RC-SEM}

In this subsection, we apply the proposed RC-SEM methodology to analyze the dataset of the Chinese shoe industry. In our empirical model, the outcome variable $Y_{i j}$ is the logarithm of the deflated prices for the $i$ th authentic firm's high-end product at year $j$. The explanatory variable of main interest, $X_{i j}$, is the quantity of counterfeit products in the market faced by the $i$ th authentic brand at year $j$, divided by the sale quantity of this authentic firm. The covariate $U$ in Equation (1) includes firm dummies and $W$ includes year dummies. The year dummies capture the effects of common shocks to the market that may vary by time. The firm dummies capture the effects of unobserved firm-level time-constant characteristics.

The entry of counterfeits is likely to be endogenous due to unobserved time-varying firm characteristics. Ignoring the endogeneity, when present, will lead to erroneous inference about its causal effect on the authentic firms' pricing. To identify the effects of the counterfeit entry, we adopt the same IV strategy as in Qian (2008). Qian (2008) documents a natural experiment in which the exogenous shocks led to the loosening of the Chinese government's monitoring of footwear trademarks. The identification strategy exploits the interaction between the unexpected enforcement change and the relationship between each branded company and the government, as proxied by the number of days it took each company to pass the required International Standards (ISO) applications. This IV strategy recognizes that branded companies that have better relationships with the government are less affected by the sudden loosening of trademark enforcements, and hence face less threats by counterfeit entry. The identification strategy uses variations in these IVs to tease out the exogenous components of the counterfeit sales which is then used to identify the causal 
effect of counterfeit entry. More institutional details regarding the IV validity are discussed in Qian (2008). In Equation (3), we use LOOSE, RELATION and LOOSE*RELATION as the main instruments, where LOOSE is an indicator variable denoting the loosening of Chinese government enforcement in monitoring the footwear trademarks, RELATION denotes the number of days it took the company to pass the required ISO applications, and $L O O S E * R E L A T I O N$ is the interaction between these two variables.

Authentic firms respond to their own counterfeit entry with various time lags. As a result, the change in prices of their products are manifested in the data only after the response time. In the analysis below, we will use RC-SEM to explicitly model the latent heterogeneous response times among the authentic firms. One advantage of the RC-SEM is that it automatically detects the presence and location of firm-specific changepoint in the outcome variable time-series. For comparison purposes, we also fit three nested models of the RC-SEM. The first one is the OLS with a pre-specified response time common to all firms. This model specification recognizes the dynamic effects of the counterfeit entry, and thus it is more realistic than a static OLS model that assumes time-invariant effect of counterfeit sales. However, this analysis requires researchers to specify a common value of response times and as a result ignores the potential heterogeneity in response times among firms. In our analysis, we pre-specify the common response time to be two years, which is the value closest to the posterior mean of response times estimated from our RC-SEM shown later. The second one is a random-changepoint $(\mathrm{RC})$ model. This model recognizes the heterogeneous response time but ignores the endogeneity issue. The third one is the simultaneous equation model (SEM) with a pre-specified response time common to all firms. The standard SEM accounts for the endogeneity issue. However, like OLS, SEM also assumes common response time with a value of two years.

We first fit the models in which the covariate $Z_{i}$ in Equation (8) contains only the 
intercept. The analysis enables us to compare the performance of different models in a relatively simple setting. All model fittings run the Gibbs sampler for 50,000 iterations and discard the first 10,000 iterations as the burn-in period. We check the convergence of the Markov chains to make sure that the chain converges to the stationary distributions after the burn-in period via both visual traceplot inspections and the Geweke's numeric diagnostic statistics (Geweke 1992).

The posterior means and standard deviations of the parameter draws from the Gibbs sampler for all models are reported in Table 1. Under the RC-SEM, we are able to detect the presence of response times that are heterogeneous among the authentic firms. Figure 3 (a) and (b) plots the prior and posterior distributions of the latent response time $\tau_{i}^{*}$. As shown in the figure, data provide a substantial amount of information so that the posterior distribution of $\tau_{i}^{*}$ is a much more condensed distribution as compared with its prior distribution. The average response time is estimated to be 1.6 years with a standard deviation of 1.1. This shows that the authentic firms took considerably different amounts of time to design and implement counter measures against their counterfeits. In contrast, neither the OLS nor SEM provides the estimates of the response times since both methods pre-specify them to be a common value of two years.

The results also show that the effect estimates of counterfeit sales are also different for different methods. Because the covariate $Z_{i}$ in Equation (8) contains only the intercept, the parameter estimates in $\Pi$ are the population mean effects. As shown in Table 1, RC-SEM shows that there is a negative (-0.34) short-term population mean effect and a positive (1.61) long-term population mean effect of counterfeit entry on the authentic firms' prices. The 95\% credible intervals for both effects exclude zero. In comparison, the results from SEM show attenuated effects for both the short-term and long-term effects. In particular, the 95\% credible interval for the short-term effect under the SEM includes zero. This shows 
that ignoring the heterogeneous response times, like what a standard SEM does, can lead to attenuated effect estimates and lose power to detect a change in the marketing response. RC and OLS also yield different estimates of the effect estimates since they do not model the endogeneous entry or heterogeneous response times. ${ }^{4}$ Table 1 shows a negative value of the posterior mean of the covariance term $\sigma_{12}$. Figure $3(\mathrm{c})$ and $(\mathrm{d})$ plot the prior and posterior distributions of the correlation coefficient, $\rho=\frac{\sigma_{12}}{\sqrt{\sigma_{11} \sigma_{22}}}$, and show that data provide strong evidence for the presence of a negative correlation, as compared with its prior distribution. This implies that there were some unobserved factors that affected the price and counterfeit entry in opposite directions. These factors could be a firm's managerial skills that are positively correlated with product prices and negatively correlated with the counterfeit entry. It is also possible that higher prices are associated with higher quality products that are harder to be imitated or counterfeited. The endogeneity issue, if not accounted for in the modeling, will lead to inconsistent estimate of causal effects.

We also conduct model comparisons using the logarithm of Bayes factors (Newton and Raftery 1994). In our model comparisons, the RC-SEM is considered as the full model, and the other three models (SEM, RC and OLS) can be considered as various nested models of the RC-SEM. ${ }^{5}$ A general rule is that a log Bayes factor of larger than 5 provides strong evidence against the null model (Raftery 1996). Our calculation shows that the log Bayes factor for RC-SEM against SEM (null model) is 350.49 which provides overwhelming evidence for the presence of heterogeneous random changepoints among firms. The log Bayes factor for RC-SEM against RC (null model) is 121.78, which provides very strong evidence for the presence of endogeneity of counterfeit entry. The log Bayes factor for RC-SEM against OLS

\footnotetext{
${ }^{4}$ In particular, both models significantly underestimate the long-term effect of counterfeit entry. Furthermore, as shown in the next subsection, these two models also mis-assess the effects of several factors on marketing outcomes and are not able to identify their significant effects.

${ }^{5}$ Because the RC and OLS use a single-equation approach, in order to make their marginal likelihood comparable with those of the SEM-type models, we have added the contribution of the likelihood from an independent model for $X$ in the calculation of marginal likelihood for these two models.
} 
(null model) is 398.63, which provides overwhelming evidence for the simultaneous presence of both heterogeneous random changepoints among firms and the endogeneity of counterfeit entry.

\subsection{Investigating Factors Affecting Firms' Responses}

In this subsection, we conduct a finer analysis to study factors affecting authentic firms' responses to the counterfeit entry. The firms' response behaviors studied here include both their response magnitude in short term and long term as well as their response speed. Specifically, we expand the RC-SEM model as specified above and include a set of observed firm characteristics to explain the differential responses in the between-firm model as specified in Equation (8). The descriptive statistics of the firm characteristics included in $Z$ are summarized in Table 2. All these variables take on the average of pre-entry values, and our analysis is concerned with how various characteristics of the firms associate with their responses to counterfeiting. In Table 3 we report estimates of the hierarchical parameters in $\Pi$ for all models considered. The covariates in $Z$ are standardized before entering the hierarchical model analysis for ease of interpretation. We will further explain these firm-level variables and the corresponding results in the following paragraphs.

The authentic product quality could moderate the counterfeiting effects in a subtle way. Our hypothesis is that it will be easier for consumers to detect counterfeits from authentic products when the authentic quality is higher. Since it is harder for counterfeiters to close the quality gap, the short-term shock to the authentic branded company will be less severe. The analyses do show that the authentic quality, as proxied by unit product costs, helps to alleviate the negative impacts of counterfeit entry on prices in the short run (Column 2 of Table 3).

Market share is an obvious brand characteristics to consider as an influencing factor. On 
the one hand, a brand with a larger market share is likely to attract more counterfeiting and face more competition that way. On the other hand, larger market share also implies the brand enjoys more market power and stability, and can be less influenceable by fringe competitions. Under our analyses of identifying the effects of plausibly exogenous entries, it does appear that brands with larger market shares are less affected by counterfeiting, with a less negative short-term effect and a smaller long-term entry effect by counterfeits (Column 3 in Table 3).

To test whether the degree of diversification moderates the effects of counterfeiting, we collected data on the number of sub-brands and percentage of the sales values for exports each branded company had. There is no significant effect associated with the number of sub-brands a branded company owns, possibly because counterfeiters infringe on all subbrands (Column 4 in Table 3). However, companies with larger percentage of sales value for export are less affected by the entry of counterfeits, because they are more diversified than the companies that rely primarily on the domestic market where counterfeits massively entered. They correspondingly have less urgency to respond to counterfeiting and have a longer response time (Column 5 in Table 3).

In theory, the more innovativeness a company is, the faster it will come up with newer product designs and innovations to differentiate from counterfeits. We use two alternative proxies for innovativeness: annual R\&D expenditures and patent application costs of each branded company. R\&D expenditures measure more of the inputs to innovation while patent costs proxy for innovation outputs (Qian 2007). These two variables are highly correlated (correlation coefficient $=0.97)$, so we include only the patent costs in the main specifications. Robustness checks using R\&D instead of patent costs yield similar results. As expected, the companies with higher levels of patent costs or R\&D responded to counterfeiting in a shorter time-frame by introducing a higher priced high-end shoes, as compared to companies with 
lower levels of innovativeness (Column 6 in Table 3). This suggests that the innovative companies not only innovate faster in the face of competition, but also innovate with better products given that Qian (2008) has shown very high correspondence between these shoe prices and their unit product costs as well as characteristics.

We additionally have information on the annual advertising expenditure of each branded company. While heavier advertising could imply that consumers are more familiar with the branded products and possibly less confused by counterfeits, it could also present a larger brand premium for counterfeiters to free ride on. It then becomes an empirical question whether advertising moderates the effect of entry by counterfeiters. Column 7 of Table 3 show that companies that are accustomed to heavier advertising raise their high-end product prices more after they introduced new products to countervail counterfeits, potentially because there is a higher brand premium to leverage or because there is a higher fixed cost to recuperate via higher prices.

Finally, we gathered data on human capital within companies to test whether and how this factor moderates the effects of counterfeiting and brand responses. We include the employment and total annual wages of the branded companies to proxy for brand-level human resources. Wage is a proxy for skilled labor commonly used in the labor economics literature. Columns 8 and 9 in Table 3 demonstrate that the more human resources a branded company has, the shorter time it takes to respond to counterfeits by innovating.

One important thing to note in the analysis is that the traditional SEM and OLS method do not model the heterogeneous response times and therefore do not allow for the study of what affects firms' reaction speed. Furthermore, ignoring the heterogeneous response time also lead to mis-assessment of the effects of firm characteristics. The RC model allows for this feature but does not model the endogeneity issue. Their effect estimates are also different from those from the RC-SEM. 


\section{A Simulation Study}

In this section, we conduct a set of simulation experiments to evaluate the performance of different models to estimate the dynamic effects in repeated samples. We follow the steps below to simulate data with dynamic effects:

- For each simulation dataset, we set the number of units $n=30$ and the number of observations per unit $J=12$, similar to those in the Chinese Shoe Market Data.

- To simulate data for $Y_{i j}, X_{i j}^{*}$ and $X_{i j}$, we first set the mean parameters in the following equations:

$$
Y_{i j}= \begin{cases}X_{i j} \beta_{1 i}+\alpha_{i}+W_{i j}^{T} \gamma+\epsilon_{i j}^{Y} & j=1, \ldots, T_{i}-1 \\ X_{i j} \beta_{2 i}+\alpha_{i}+W_{i j}^{T} \gamma+\epsilon_{i j}^{Y} & j=T_{i}, \ldots, J\end{cases}
$$

and

$$
\begin{aligned}
& X_{i j}^{*}=\delta Z_{i j}+\epsilon_{i j}^{X} \\
& X_{i j}= \begin{cases}X_{i j}^{*} & \text { if } X_{i j}^{*} \geq 0 \\
0 & \text { if } X_{i j}^{*}<0 .\end{cases}
\end{aligned}
$$

In simulations, we set the short-term effect $\beta_{1}=-0.4$ and the long-term effect $\beta_{2}=1.0$. The parameter vector $\gamma$ includes the time fixed-effects that are simulated from $N\left(0,0.1^{2}\right)$. The firm effects $\alpha_{i}$ is simulated from $N\left(0,0.1^{2}\right)$. In simulations, we include in $Z$ an intercept and three instrumental variables. The first IV is 0 before $J=4$ and 1 afterward, mimicking the occurrence of a natural experiment. The second IV is simulated from $\mathrm{N}(0.5,1)$, and the third IV is the interaction of the first two IVs. We set the parameter values for coefficients on the intercept and the IVs to be $(-1,2,0,0.5)$. We then randomly generated the response time $\tau_{i}^{*}$ from $N\left(2,1.5^{2}\right)$ and form the grouped version of the response time $\tau_{i}$ according to Equation (5). The changepoint time $T_{i}=T_{i 0}+\tau_{i}$, where $T_{i 0}$ is the first time that the treatment variable becomes positive. 
- We generate the error terms $\left(\epsilon_{i j}^{X}, \epsilon_{i j}^{Y}\right)$ from a bivariate normal distribution with mean 0 and variance-covariance matrix

$$
\Sigma_{\epsilon}=\left(\begin{array}{cc}
\sigma_{11} & \rho \sqrt{\sigma_{11} \sigma_{22}} \\
\rho \sqrt{\sigma_{11} \sigma_{22}} & \sigma_{22}
\end{array}\right)
$$

where $\rho$ is the correlation coefficient of two error terms. In simulations, we set $\sigma_{11}=0.15^{2}$ and $\sigma_{22}=0.5^{2}$, and we vary $\rho$ in $(0,-0.2,0.2,-0.5,0.5,0.8,-0.8)$ to cover various strengths of endogeneity in both directions.

- We then generate $Y_{i j}, X_{i j}^{*}$ and $X_{i j}$ given the above model parameters. We repeat the above steps for $M=50$ times for each parameter setting. This will generate $7^{*} M$ panel datasets because there are seven values of $\rho$ specified in step 3 .

- We fit each simulated dataset with four models: the RC-SEM, SEM, RC model and the OLS model. Note that RC-SEM model is the full model and the other models can be considered as reduced models of RC-SEM. The RC model assumes $\rho=0$ (i.e. no endogeneity issue) but allows heterogeneity of response times across units. In both SEM and the OLS the response times are assumed to be the same for all units. Furthermore, this common response time is not to be estimated from the data in SEM and OLS, but rather needs to be pre-specified. In the simulation study, we assume this common response time is 2 , which is the mean of the response time used in the simulation. This corresponds to a scenario that the best guess of the response time, under the assumption of common response time, is used.

- We compare the estimates for both the population temporary short-term effect $\beta_{1}$ and the stable long-term entry effect $\beta_{2}$ from these four models. The result is summarized in Table 4. The columns "Bias", "SD" and "RMSE" are the bias, standard deviation, and square root of Mean squared error, respectively, calculated from the resulting sample of Bayesian 
estimates. We repeat the process for each value of $\rho$. The column "Coverage Rate" is the proportion of $95 \%$ credible intervals that contain the true values in the simulation experiments.

The simulation study shows that the estimation algorithm under the RC-SEM model recovers the true values of the temporary short-term $\beta_{1}$ and stable long-term entry effect $\beta_{2}$ reasonably well. Its RMSEs are smallest among four models across different strength of endogeneity. In addition, the coverage rates of the credible intervals are closest to the nominal 95\% rate, among all methods. The simulation study shows that both the SEM model and OLS that ignores the heterogeneity in latent response times attenuate dynamic entry effects. The attenuation bias could be as large as $50 \%$ reduction in the true effect size. This shows that in the presence of heterogeneous response time, ignoring the heterogeneity and specifying a common response time can lead to severely biased estimates of dynamic effects. Moreover, the Bayesian estimator from the RC-SEM model has less variability (i.e. smaller standard error) than that from the SEM, because the RC-SEM model provides better model-fitting by taking into account the latent response times. The RC estimates are biased because of the endogeneity issue. The OLS estimate has serious bias, particulary when the endogeneity is strong, and the $95 \%$ credible intervals hardly contain the true effect value.

\section{Discussion}

In marketing and economics studies, measuring the causal relationships among variables is of critical importance. In practical applications, however, the issues of nonstationary, endogeneity and heterogeneity are frequently encountered and can potentially spoil inference if not taken into account properly. In this paper, we have considered the modeling and inference of a simultaneous equation model with random changepoints, and apply the model to study the dynamic and heterogeneous causal effects of counterfeit entry on the authentic 
firm's price. The set of incumbent responses to entry by counterfeiters provide an interesting case study to demonstrate the applicability of the proposed methodology. To the best of our knowledge, this is the first study to propose and apply a tractable framework to account for all the above issues. As shown in our application and simulations, because the proposed method considers both the heterogeneous nature of marketing players' response times and the endogeneity issue, it minimizes the bias in the dynamic effect estimation. These more accurate dynamic effect estimates are also beneficial for future welfare analyses and policy experiments.

An added benefit of our method is the automatic detection of changepoints across units and the data-driven estimations of such changepoints (i.e., the authentic companies' response times in our application), which are interesting to study in their own rights. We further study what affects the firms' response times along with their response magnitudes using the hierarchical Bayesian method. We find that firms that have more human resources or less diversification from markets affected by trademark infringement are quicker to fire sustainable long-term responses to new competitive threats by counterfeiters. As demonstrated in the application, the proposed method provides marketing researchers with a new approach to study similar questions on response times using field data.

The empirical results in this paper help to unify two strands of Industrial Organization literature on the entry effects on prices. In particular, the finding that the authentic prices fell immediately upon the entry of counterfeiters can be explained by Fudenberg and Tirole (2000). That is, new entry imposes competitive pressure in the short-run. We further identify that authentic prices rose substantially on average two years after counterfeit entry. This positive effect could be resolved with the other strand of theories. Notably, Frank and Salkever (1997) predicts that generic entry could steal away the price-sensitive consumer segment, leaving behind a more inelastic demand for the branded companies to re-optimize into 
a higher price. In addition, Qian (2006) predicts that companies invest to differentiate their products from counterfeits through innovation, self-enforcement, vertical integration, as well as price signaling, and all these mechanics lead to price increases. It becomes apparent that these theories can better explain the long-term entry effects, and are complementary rather than contradictory to traditional economic theories that predict negative price shocks. The individual-level parameter estimates also uncover a set of firm characteristics that moderate the timing and magnitude of pricing responses to entry by counterfeiters. These results prescribe effective brand management strategies tailored to each type of firms. The empirical findings on the pricing effects of counterfeiting can also shed lights on the private label literature, where imitation or copycat strategy accounts for more than $50 \%$ of the store brand introductions (Scott Morton and Zettelmeyer, 2004).

There are also some possible extensions to the proposed model. Our modeling framework is well suited for studying two-stage dynamic effects with a temporary short-term effect and a stable long-term effect. Such a model provides a relatively parsimonious summarization of the dynamic effects of market changes on the responses. In other settings (e.g. FHH 2004), each study unit may have multiple changepoints in their panel responses. One could extend the model developed in this article to allow for such scenario. Second, our hierarchical model uses the standard multivariate normal model for unit-specific latent variables. One could exploit the usage of a more general second stage model, such as a multivariate- $t$ distribution. In summary, the method proposed in this article is flexible and has potential to be expanded along these directions.

\section{References}

Amemiya, T. (1985) "Advanced Econometrics," Cambridge, MA: Harvard University Press. Bai, J (1997), "Estimation of a Change Point in Multiple Regression Models," Review of 
Economics and Statistics, 79, 551-563.

Barry, D. and Hartigan, J.A. (1993), "A Bayesian Analysis for Change Point Problems," Journal of the American Statistical Association, 88, 309-319.

Böckenholt, U. and Dillon W.R. (1997). "Some New Methods for an Old Problem: Modeling Preference Changes and Competitive Market Structures in Pretest Market Data." Journal of Marketing Research, 15, 130-142.

Bowman, D., H. Gatignon (1995). "Determinants of competitor response time to a new product introduction." Journal of Marketing Research. 32 (February): 4253.

Carlin, B., Gelfand, A.E. and Smith, A.F.(1992), "Hierarchical Bayesian Analysis of Changepoint Problems," Applied Statistics, 41, 389-405.

Carlton D. and Perloff J. M. (2005), Modern Industrial Organization, 2nd Edition, Pearson/Addison Wesley.

Chib, S. (1998) "Estimation and comparison of multiple change-point models," Journal of Econometrics, 86, 221-241

Choate, P. (2005). Hot Property : The Stealing of Ideas in an Age of Globalization. Westminster, MD, USA: Alfred A. Knopf Incorporated, 2005.

Deleersnyder, Barbara, Marnik G. Dekimpe, Jan-Benedict Steenkamp and Oliver Koll (2007), "Win-win Strategies at Discount Stores", Journal of Retailing and Consumer Services, 14(5), 309-18.

Fader, P.S., Hardie, B.G.S. and Huang, C.Y. (2004), "A Dynamic Changepoint Model for New Product Sales Forecasting,", Marketing Science, 23, 50-65.

Fong, D.K.H. and DeSarbo, W.S. (2007), "A Bayesian Methodology for Simultaneously Detecting and Estimating Regime Change Points and Variable Selection in Multiple Regression Models for Marketing Research,", Quantitative Marketing and Economics, 5, $427-453$.

Frank, R.G. and Salkever, D.S. (1997), "Generic Entry and the Pricing of Pharmaceuticals", Journal of Economics \& Management Strategy, 6:1, 75-90.

Fudenberg, D. and Tirole, J. (2000), "Pricing a Network Good to Deter Entry", The Journal of Industrial Economics, 48:4, 373-390.

Geweke, J. (1992), Evaluating the Accuracy of Sampling-based Approaches to Calculating Posterior Moments, Bayesian Statistics 4, Eds: Bernado, J.M., Berger, J.O., Dawid, A.P. and Smith, A.F.M., Clarendon Press: Oxford.

Heil, O., and Robertson, T.S. (1991), "Toward a Theory of Competitive Market Signaling: A Research Agenda," Strategic Management Journal, 12, 403-418.

Hoch, Stephen and Shumeet Banerji (1993), "When Do Private Labels Succeed?" Sloan Management Review34(4), 57-67. 
Kotler, P. (1971), "Marketing Decision Making: A Model Building Approach," Holt, Rinehart \& Winston.

Lahari, K and Schmidt, P. (1978), "On the Estimation of Triangular Structural models," Econometrica,46, 1217-1221.

Neelamegham, R., and Chintagunta, P.K. (2004), "Modeling and Forecasting the Sales of Technology Products," Quantitative Marketing and Economics,2, 195-232.

Newton, M. and Raftery, A. (1994) "Approximate Bayesian Inference by the Weighted Likelihood Bootstrap," Journal of the Royal Statistical Society, Series B, 56, 3-48.

Pauwels, Koen and Shuba Srinivasan (2004), "Who Benefits From Store Brand Entry?", Marketing Science, 23(4), 596-610.

Poulsen, C.S. (1990), "Mixed Markov and Latent Markov Modeling Applied to Brand Choice Behavior," International Journal of Research in Marketing,7, 5-19.

Qian, Y. (2006), "Pricing and Marketing Effects of Entry by Imitators or Counterfeits", Harvard Working Paper.

Qian, Y. (2007), "Do National Patent Laws Stimulate Domestic Innovation in a Global Patenting Environment? A Cross-Country Analysis of Pharmaceutical Patent Protection, 1978-2002," The Review of Economics and Statistics, 89(3), 436-453

Qian, Y. (2008), "Impacts of Entry by Counterfeiters," Quarterly Journal of Economics, 123(4), 1577-1609.

Raftery, A. E. (1996), Hypothesis Testing and Model Selection, Markov Chain Monte Carlo in Practice, Eds: Gilks, W.R., Richardson, S. and Spiegelhalter, D.J., Chapman \& Hall: London.

Ramaswamy, V. (1997). "Evolutionary Preference Segmentation with Panel Survey Data: An Application to New Products," International Journal of Research in Marketing. 14: $57-80$.

Rao, Akshay R. and Kent B. Monroe (1996), "Causes and Consequences of Price Premiums", Journal of Business, 69, 511-35.

Robinson, W. T. (1988). "Marketing Mix Reactions to Entry." Marketing Science. 7 (4): $368-385$.

Rossi, P., Allenby, G.M. and McCulloch, R.E. (2005), Bayesian Statistics and Marketing, Hoboken, NJ: Wiley.

Schweidel, D.A. and Fader, P.S. (2009), "Dynamic Changepoints Revisited: An Evolving Process Model of New Product Sales ," International Journal of Research in Marketing, forthcoming. 
Scott Morton, Fiona and Florian Zettelmeyer (2004), "The Strategic Positioning of Store Brands in Retailer-manufacturer Negotiations", Review of Industrial Organization, 24, 161-94.

Smith, A.F.M (1975), "A Bayesian Approach to Inference about a Change-point in a Sequence of Random Variables ," Biometrika, 62, 407-416.

Smith, K.G., Grimm, C.M., Chen, M., and Gannon, M. J. (1989), "Predictors of Response Time to Competitive Strategic Actions: Preliminary Theory and Evidence," Journal of Business Research, 18, 245-258.

Tanner, M.A. and Wong, W.H. (1987), "The Calculation of Posterior Densities by Data Augmentation (with discussion)," Journal of the American Statistical Association, 82, $528-550$.

Van Heerde, H.J., Mela, C.F., and Manchanda, P. (2004), "The Dynamic Effect of Innovation on Market Structure," Journal of the Marketing Research, XLI, 166-183.

World Customs Organization (2004), "Counterfeiting Congress Calls for Public-private Cooperation". http://www.wcoomd.org/ie/En/Press/press.html, May 25, 2004. 


\section{Appendix: Prior Specification and MCMC algorithm for the RC-SEM.}

In this Appendix, we present the details of prior specification and the MCMC algorithm for estimating the proposed simultaneous equation model with random changepoints (RC-SEM).

Equation (10) presents the forms of the priors. In our analysis, we set the constants in the priors as follows: $\mu_{\Pi}, \mu_{\gamma}, \mu_{\delta}$ are assigned as vectors of zeros. $\Lambda_{\Pi}=0.01 \times I_{n_{r} \times n_{z}}$, $A_{\gamma}=0.01 \times I_{n_{\gamma}}, A_{\delta}=0.01 \times I_{n_{\delta}}, \nu_{\epsilon}=5$ and $\nu_{e}=n_{r}+3, S_{\epsilon}=\nu_{\epsilon} \Sigma_{\epsilon 0}$ and $S_{e}=\nu_{e} \Sigma_{e 0}$, where $n_{r}$ is the dimension of the square matrix of $\Sigma_{e}$, and $n_{z}$ is the number of columns of $Z, n_{\gamma}$ and $n_{\delta}$ is the length of $\gamma$ and $\delta$.

When choosing the value for $\Sigma_{\epsilon 0}$ and $\Sigma_{e 0}$, we follow the approach suggested in Rossi et al. (2005). Because $\Sigma_{\epsilon 0}$ is related to the mean of the prior for the variance-covariance matrix of the error terms of the simultaneous equation model, we would like to take into account the scale of the outcomes and the explanatory power of the regressors in the assignment of its value. Rather than assigning an arbitrary value, such as an identity matrix, we set $\Sigma_{\epsilon 0}=\hat{\Sigma}_{\epsilon}^{O L S}$, where the diagonal entries of $\hat{\Sigma}_{\epsilon}^{O L S}$ are OLS estimates of error variances for $Y$ equation and $X$ equation, separately, and the off-diagonal entries of $\hat{\Sigma}_{\epsilon}^{O L S}$ are zeros. The choice of the prior is reasonable because the resulting prior is reasonably flat over a wide range of plausible values of the correlation coefficient $\rho$ between $\epsilon^{X}$ and $\epsilon^{Y}$, the measure of the endogeneity strength. Figure 3 (c) displays the marginal prior distribution of the correlation coefficient $\rho=\frac{\sigma_{12}}{\sqrt{\sigma_{11} \sigma_{22}}}$ given the choices of the above chosen prior. The histogram is constructed from sampling 10000 iid draws from the priors of $\Sigma_{\epsilon}$, and then calculating $\rho$ for each draw. The histogram shows that for this prior, the distribution of correlation coefficient $\rho$ is centered at zero and reasonably spread out between -1 and +1 . Similarly, we have set the block in $\Sigma_{e 0}$ for $\left(\alpha_{i}, \beta_{1 i}, \beta_{2 i}\right)$ as $\hat{\Sigma}_{e}^{O L S}$. Because the OLS does not model the heterogeneity of $\tau_{i}^{*}$, we set the corresponding entry in $\Sigma_{e 0}$ to be one. Using a larger value ( e.g., 2) or a smaller value (e.g., 0.5) has little impact on the resulting estimation. 
Given the above prior and posterior distribution derived in Equation (11), we implement a Gibbs sampler to obtain draws from the posterior distribution. The full conditionals of model unknowns for each step of the Gibbs sampler are derived below.

1. Draw $\beta_{1 i}, \beta_{2 i}, \alpha_{i} \mid \delta, \gamma, \Sigma_{\epsilon}, T_{i}, X^{*}$

We decompose the joint bivariate normal distribution of the error term $\left(\epsilon_{i j}^{X}, \epsilon_{i j}^{Y}\right)$ as the product of the marginal distribution of $\epsilon_{i j}^{X}$ and the conditional distribution of $\epsilon_{i j}^{Y} \mid \epsilon_{i j}^{X}$. We note that the conditional distribution $\epsilon_{i j}^{Y} \mid \epsilon_{i j}^{X}$ is $N\left(\frac{\sigma_{12}}{\sigma_{22}} \epsilon_{i j}^{X}, \sigma_{11}-\frac{\sigma_{12}^{2}}{\sigma_{22}}\right)$. We write $\epsilon_{i j}^{Y}=\frac{\sigma_{12}}{\sigma_{22}} \epsilon_{i j}^{X}+e_{i j}$, where $e_{i j} \sim N\left(0, \sigma_{e}^{2}\right), \sigma_{e}^{2}=\sigma_{11}-\frac{\sigma_{12}^{2}}{\sigma_{22}}$ and $e_{i j} \perp \epsilon_{i j}^{X}$. Given $\delta$, the error term $\epsilon_{i j}^{X}=X_{i j}^{*}-\delta Z_{i j}$. To make notation more compact at the unit level, let $Y_{i}=\left(Y_{i 1}, \ldots, Y_{i J}\right), \epsilon_{i}^{X}=\left(\epsilon_{i 1}^{X}, \ldots, \epsilon_{i J}^{X}\right), e_{i}=\left(e_{i 1}, \ldots, e_{i J}\right)$. Then at the unit level, we have:

$$
Y_{i}-W_{i}^{T} \gamma-\frac{\sigma_{12}}{\sigma_{22}} \epsilon_{i j}^{X}=H_{i}\left[\begin{array}{c}
\beta_{1 i} \\
\beta_{2 i} \\
\alpha_{i}
\end{array}\right]+e_{i}
$$

where the design matrix for the $i$ th unit $H_{i}$ is:

$$
H_{i}=\left(H_{i 1}^{T}, \ldots, H_{i J}^{T}\right)^{T}=\left[\begin{array}{lll}
X_{i 1} & 0 & U_{i 1} \\
\cdot & \cdot & \cdots \\
X_{i, T_{i}-1} & 0 & \ldots \\
0 & X_{i, T_{i}} & \ldots \\
\cdot & \cdot & \ldots \\
0 & X_{i, J} & U_{i J}
\end{array}\right],
$$

and $T_{i}=T_{i 0}+\tau_{i}$. We rewrite the variance-covariance matrix, $\Sigma_{e}$, in the distribution of the latent variables $\left(\beta_{1 i}, \beta_{2 i}, \alpha_{i}, \tau_{i}^{*}\right)$ in Equation (9) as follows,

$$
\Sigma_{e}=\left[\begin{array}{ll}
\Sigma_{e, 11} & \Sigma_{e, 12} \\
\Sigma_{e, 12} & \Sigma_{e, 22}
\end{array}\right]
$$

where $\Sigma_{e, 11}$ and $\Sigma_{e, 22}$ are variance-covariance matrix of $\left(\beta_{1 i}, \beta_{2 i}, \alpha_{i}\right)$ and $\tau_{i}^{*}$, respectively, and $\Sigma_{e, 12}$ is covariance matrix between these two blocks of parameters. The prior 
distribution of $\left(\beta_{1 i}, \beta_{2 i}, \alpha_{i}\right) \mid \tau_{i}^{*}$ is $N\left(\mu_{1 \mid 2, i}, A_{1 \mid 2}^{-1}\right)$ where

$$
\begin{aligned}
\mu_{1 \mid 2, i} & =\mu_{1 i}+\Sigma_{e, 12} \Sigma_{e, 22}^{-1}\left(\tau_{i}^{*}-\mu_{2 i}\right) \\
A_{1 \mid 2}^{-1} & =\Sigma_{e, 11}-\Sigma_{e, 12} \Sigma_{e, 22}^{-1} \Sigma_{e, 21}
\end{aligned}
$$

and $\mu_{1 i}$ and $\mu_{2 i}$ are the prior means of $\left(\beta_{1 i}, \beta_{2 i}, \alpha_{i}\right)$ and $\tau_{i}$, respectively, and $\left(\mu_{1 i}, \mu_{2 i}\right)=$ $\Pi Z_{i}$. Then the conditional draws of $\left(\beta_{1 i}, \beta_{2 i}, \alpha_{i}\right)$ can be obtained from the following normal distribution:

$$
N\left(\left(\frac{H_{i}^{T} H_{i}}{\sigma_{e}^{2}}+A_{1 \mid 2}\right)^{-1}\left(\frac{H_{i}^{T} R_{i}}{\sigma_{e}^{2}}+A_{1 \mid 2} \mu_{1 \mid 2, i}\right), \quad\left(\frac{H_{i}^{T} H_{i}}{\sigma_{e}^{2}}+A_{1 \mid 2}\right)^{-1}\right)
$$

where $R_{i}=Y_{i}-W_{i}^{T} \gamma-\frac{\sigma_{12}}{\sigma_{22}} \epsilon_{i}^{X}$.

2. $\gamma \mid \delta, \beta_{1 i}, \beta_{2 i}, \alpha_{i}, \Sigma_{\epsilon}, T, X^{*}$

Given the prior distribution for $\gamma$ as $N\left(\mu_{\gamma}, A_{\gamma}^{-1}\right)$, we have the conditional draw of $\gamma$ as

$$
N\left(\left(\frac{W_{i}^{T} W_{i}}{\sigma_{e}^{2}}+A_{\gamma}\right)^{-1}\left(\frac{W_{i}^{T} R_{i}^{\gamma}}{\sigma_{e}^{2}}+A_{\gamma} \mu_{\gamma}\right), \quad\left(\frac{W_{i}^{T} W_{i}}{\sigma_{e}^{2}}+A_{\gamma}\right)^{-1}\right),
$$

where $R_{i}^{\gamma}=Y_{i}-H_{i}\left[\beta_{1 i}, \beta_{2 i}, \alpha_{i}\right]^{T}-\frac{\sigma_{12}}{\sigma_{22}} \epsilon_{i}^{X}$.

3. $X_{i j}^{*} \mid \beta_{1 i}, \beta_{2 i}, \alpha_{i}, \gamma, \delta, \Sigma_{\epsilon}, T$

If $X_{i j}>0$, then $X_{i j}^{*}=X_{i j}$. If $X_{i j}=0$, then $X_{i j}^{*}$ is drawn from a truncated normal distribution with the mean $Z_{i j} \delta+\frac{\sigma_{12}}{\sigma_{11}} \epsilon_{i j}^{Y}$ and the variance $\sigma_{22}-\frac{\sigma_{12}^{2}}{\sigma_{11}}$, where the truncation is to $(-\infty, 0)$.

4. $\delta \mid \beta_{1 i}, \beta_{2 i}, \alpha_{i}, \gamma, \Sigma_{\epsilon}, T, X^{*}$

Following Lahari and Schmidt (1978) and Rossi et al. (2005), we re-express the triangular system specified in Equation (1), (3) and (4) as a SUR model for making 
conditional draw of $\delta$. Let $Y_{i}^{*}=Y_{i}-H_{i}\left(\beta_{1 i}, \beta_{2 i}, \alpha_{i}\right)^{T}$, where $H_{i}$ is given in Equation (12). We then have the likelihood of the triangular system the same as that of the following SUR model:

$$
\left(\begin{array}{c}
Y_{i j}^{*} \\
X_{i j}^{*}
\end{array}\right)=\left(\begin{array}{ll}
W_{i j} & \\
& Z_{i j}
\end{array}\right)\left(\begin{array}{l}
\gamma \\
\delta
\end{array}\right)+\left(\begin{array}{c}
\epsilon_{i j}^{Y} \\
\epsilon_{i j}^{X}
\end{array}\right)
$$

where $Y_{i j}^{*}$ is the $j$ th component of $Y_{i}^{*}, W_{i j}$ is the $j$ th row in the design matrix $W_{i}$. Let $M_{i j}=\left(\begin{array}{cc}W_{i j} & \\ & Z_{i j}\end{array}\right)$. Then $(\gamma, \delta)$ has a likelihood as that from a multivariate normal with the mean

$$
(\bar{\gamma}, \bar{\delta})=\left(\sum_{i, j} M_{i j}^{T} \Sigma_{\epsilon}^{-1} M_{i j}\right)^{-1}\left(\sum_{i, j} M_{i j}^{T} \Sigma_{\epsilon}^{-1}\left(\begin{array}{c}
Y_{i j}^{*} \\
X_{i j}^{*}
\end{array}\right)\right)
$$

and the variance-covariance matrix

$$
\left(\begin{array}{cc}
\Omega_{\gamma \gamma} & \Omega_{\gamma \delta} \\
\Omega_{\delta \gamma} & \Omega_{\delta \delta}
\end{array}\right)=\left(\sum_{i, j} M_{i j}^{T} \Sigma_{\epsilon}^{-1} M_{i j}\right)^{-1}
$$

Then given $\gamma$, the likelihood for $\delta$ is a multivariate normal with mean $\mu_{\delta \mid \gamma}=\bar{\delta}+$ $\Omega_{\delta \gamma} \Omega_{\gamma \gamma}^{-1}(\gamma-\bar{\gamma})$ and variance-covariance matrix $\Omega_{\delta \mid \gamma}=\Omega_{\delta \delta}-\Omega_{\delta \gamma} \Omega_{\gamma \gamma}^{-1} \Omega_{\gamma \delta}$. Thus given the a normal prior $N\left(\mu_{\delta}, A_{\delta}^{-1}\right)$ for $\delta$, we can draw $\delta$ from a normal distribution with mean $\left(A_{\delta}+\Omega_{\delta \mid \gamma}^{-1}\right)^{-1}\left(A_{\delta} \mu_{\delta}+\Omega_{\delta \mid \gamma}^{-1} \mu_{\delta \mid \gamma}\right)$ and variance $\left(A_{\delta}+\Omega_{\delta \mid \gamma}^{-1}\right)^{-1}$.

5. $\Sigma_{\epsilon} \mid \beta_{1 i}, \beta_{2 i}, \alpha_{i}, \gamma, \delta, T, X^{*}$

The conditional draw of $\Sigma_{\epsilon}$ follows $I W\left(\nu_{\epsilon}+N \times J, S_{\epsilon}+S\right)$, where $S=\sum_{i, j}\left(\begin{array}{c}\epsilon_{i j}^{Y} \\ \epsilon_{i j}^{X}\end{array}\right)\left(\epsilon_{i j}^{Y}, \epsilon_{i j}^{X}\right)$.

6. $\tau_{i}^{*}, \tau_{i} \mid \beta_{1 i}, \beta_{2 i}, \alpha_{i}, \gamma, \delta, \Sigma_{\epsilon}, X^{*}$

Given other parameters and latent data, we can calculate the vector $R_{i}=Y_{i}-$ $\frac{\sigma_{12}}{\sigma_{22}} \epsilon_{i}^{X}$. For the $i$ th response time $\tau_{i}$, we have its conditional distribution given by 
multinomial $\left(1,\left[p_{i}(0), \ldots, p_{i}\left(J-T_{i 0}+1\right)\right]\right)$, where

$$
p_{i}(m)=\frac{f\left(R_{i} \mid \tau_{i}=m\right) p\left(\tau_{i}=m\right)}{\sum_{m^{\prime}=0}^{J-T_{i 0}+1} f\left(R_{i} \mid \tau_{i}=m^{\prime}\right) p\left(\tau_{i}=m^{\prime}\right)}
$$

for $m=0, \ldots, J-T_{i 0}+1$ and $f\left(R_{i} \mid \tau_{i}=m\right)$ is a density function for $M V N\left(H_{i, m}\left(\beta_{1 i}, \beta_{2 i}, \alpha_{i}\right)^{T}+\right.$ $\left.W_{i} \gamma, \sigma_{e}^{2} I_{J}\right), \sigma_{e}^{2}=\sigma_{11}-\frac{\sigma_{12}^{2}}{\sigma_{22}}$ and $H_{i, m}$ is:

$$
H_{i, m}=\left[\begin{array}{lll}
X_{i 1} & 0 & U_{i 1} \\
\cdot & \cdot & \cdots \\
X_{i, m+T_{i 0}-1} & 0 & \cdots \\
0 & X_{i, m+T_{i 0}} & \cdots \\
\cdot & \cdot & \cdots \\
0 & X_{i, J} & U_{i J}
\end{array}\right]
$$

The prior distribution $p\left(\tau_{i}=m\right)$ is calculated from the conditional distribution of $\left(\tau_{i}^{*} \mid \beta_{1 i}, \beta_{2 i}, \alpha_{i}\right)$, using the fact that $\tau_{i}$ is grouped from the underlying continuous variables $\tau_{i}^{*}$. Thus $p\left(\tau_{i}=m\right)$ has the following probabilities

$$
p\left(\tau_{i}=m\right)= \begin{cases}\Phi\left(0 \mid \mu_{2 \mid 1, i}, A_{2 \mid 1}^{-1}\right) & \text { if } m=0 \\ \Phi\left(m \mid \mu_{2 \mid 1, i}, A_{2 \mid 1}^{-1}\right)-\Phi\left(m-1 \mid \mu_{2 \mid 1, i}, A_{2 \mid 1}^{-1}\right) & \text { if } 0<m<J-T_{i 0}+1 \\ 1-\Phi\left(m-1 \mid \mu_{2 \mid 1, i}, A_{2 \mid 1}^{-1}\right) & \text { if } m=J-T_{i 0}+1,\end{cases}
$$

where $\Phi\left(\cdot \mid \mu_{2 \mid 1, i}, A_{2 \mid 1}^{-1}\right)$ is the cumulative probability function from $N\left(\mu_{2 \mid 1, i}, A_{2 \mid 1}^{-1}\right)$ and

$$
\begin{aligned}
\mu_{2 \mid 1, i} & =\mu_{2 i}+\Sigma_{e, 21} \Sigma_{e, 11}^{-1}\left(\left(\beta_{1 i}, \beta_{2 i}, \alpha_{i}\right)^{T}-\mu_{1 i}\right) \\
A_{2 \mid 1}^{-1} & =\Sigma_{e, 22}-\Sigma_{e, 21} \Sigma_{e, 11}^{-1} \Sigma_{e, 12}
\end{aligned}
$$

and $\mu_{1 i}$ and $\mu_{2 i}$ are the prior means of $\left(\beta_{1 i}, \beta_{2 i}, \alpha_{i}\right)$ and $\tau_{i}^{*}$, respectively, and $\left(\mu_{1 i}, \mu_{2 i}\right)=$ $\Pi Z_{i}$. Given the draw of $\tau_{i}$, we can draw $\tau_{i}^{*}$ from a truncated normal from $N\left(\mu_{2 \mid 1, i}, A_{2 \mid 1}^{-1}\right)$ with the following lower, $a$, and upper bound, $b$,

$$
(a, b)=\left\{\begin{array}{cl}
(- \text { inf, } 0] & \text { if } m=0 \\
(m-1, m] & \text { if } 0<m<J-T_{i 0}+1 \\
(m-1, \text { inf }) & \text { if } m=J-T_{i 0}+1
\end{array}\right.
$$


7. Updating $\Pi, \Sigma_{e} \mid \beta_{1 i}, \beta_{2 i}, \alpha_{i}, Z_{i}$.

Let $\Lambda=\Sigma_{e}^{-1}$. We follow the standard approach (Gelman et al. 2004, Rossi et al. 2005) to obtain the conditional draws as follows:

$$
p(\Lambda \mid \Theta)=\mathrm{W}\left(\nu_{e}+N,\left(S_{e}+\sum_{i=1}^{N} e_{i} e_{i}^{\prime}\right)^{-1}\right),
$$

where $e_{i}=\beta_{i}-\Pi Z_{i}, \beta_{i}=\left(\alpha_{i}, \beta_{1 i}, \beta_{2 i}, \tau_{i}\right)$, and

$$
p(\Theta \mid \Lambda)=\mathrm{N}\left(\Sigma_{\Pi}\left[\left(\Lambda \otimes I_{n_{z}}\right) H_{z \beta}+\Lambda_{\Pi} \mu_{\Pi}\right], \Sigma_{\Pi}\right)
$$

$N$ is the number of subjects, $\Sigma_{\Pi}=\left[\left(\Lambda \otimes H_{z z}\right)+\Lambda_{\Pi}\right]^{-1}$, and

$$
H_{z z}=\sum_{i=1}^{N} Z_{i} Z_{i}^{\prime}, \quad H_{z \beta}=\left[\begin{array}{c}
H_{z \beta_{1}} \\
H_{z \beta_{2}} \\
\vdots \\
H_{z \beta_{n_{r}}}
\end{array}\right],
$$

and $H_{z \beta_{j}}=\sum_{i=1}^{N} Z_{i} \beta_{i j}$, for $j=1, \ldots, n_{r}$. 
Table 1: Estimation Results Under RC-SEM and Reduced Models When $Z$ Contains Only Intercept.

Note: The table lists the posterior means and standard deviations of model parameters. $\log (d f p h)$ : the logarithm of deflated authentic high-end prices in Chinese Yuan, using the Consumer Price index published in the World Bank World Development Indicators (WDI) (Year 1995 was set as the base year in the database, i.e. CPI=100 in 1995). fksh: the quantity of counterfeit products in the market faced by the corresponding authentic firm, divided by the sale quantity of this authentic firm. $f k s h_{S T}$ and $f k s h_{L T}$ refer to its short-term and long-term effects, respectively. RC-SEM: the simultaneous equation model with random changepoints. SEM: the standard simultaneous equation model with a common response time of two years. RC: randomchangepoint model. OLS: the standard OLS model with a common response time of two years. All models use year fixed effects. "*" indicates that $95 \%$ credible interval excludes zero.

\begin{tabular}{|c|c|c|c|c|c|c|}
\hline \multirow{2}{*}{ Explanatory Variable } & \multicolumn{2}{|c|}{ RC-SEM } & \multicolumn{2}{|c|}{ SEM } & \multirow{2}{*}{$\frac{\mathrm{RC}}{\log (d f p h)}$} & \multirow{2}{*}{$\frac{\text { OLS }}{\log (d f p h)}$} \\
\hline & $\log (d f p h)$ & $f k s h$ & $\log (d f p h)$ & $f k s h$ & & \\
\hline \multirow[t]{2}{*}{ CONST } & $1.36^{*}$ & & $1.34^{*}$ & & $1.41^{*}$ & $1.36^{*}$ \\
\hline & $(0.12)$ & & $(0.10)$ & & $(0.11)$ & $(0.11)$ \\
\hline \multirow[t]{2}{*}{$\mathrm{fksh}_{S T}$} & $-0.34^{*}$ & & -0.05 & & $-0.57^{*}$ & $-0.24^{*}$ \\
\hline & $(0.08)$ & & $(0.17)$ & & $(0.10)$ & $(0.07)$ \\
\hline \multirow[t]{2}{*}{$\mathrm{fksh}_{L T}$} & $1.61^{*}$ & & $1.02^{*}$ & & $1.24^{*}$ & $0.75^{*}$ \\
\hline & $(0.25)$ & & $(0.24)$ & & $(0.27)$ & $(0.11)$ \\
\hline \multirow[t]{2}{*}{ ResponseTime } & $1.57^{*}$ & & NA & & $1.65^{*}$ & NA \\
\hline & $(0.11)$ & & NA & & $(0.13)$ & NA \\
\hline \multirow[t]{2}{*}{ LOOSE } & & $0.59^{*}$ & & $0.53^{*}$ & & \\
\hline & & $(0.10)$ & & $(0.09)$ & & \\
\hline \multirow[t]{2}{*}{ RELATION } & & 0.000 & & 0.001 & & \\
\hline & & $(0.001)$ & & $(0.001)$ & & \\
\hline \multirow[t]{2}{*}{ LOOSE*RELATION } & & $0.003^{*}$ & & $0.003^{*}$ & & \\
\hline & & $(0.001)$ & & $(0.001)$ & & \\
\hline \multirow[t]{2}{*}{$\sigma_{11}$} & \multirow{2}{*}{\multicolumn{2}{|c|}{$\begin{array}{l}0.01^{*} \\
(0.001)\end{array}$}} & \multirow{2}{*}{\multicolumn{2}{|c|}{$\begin{array}{l}0.02^{*} \\
(0.002)\end{array}$}} & $0.01^{*}$ & $0.02^{*}$ \\
\hline & & & & & $(0.001)$ & $(0.002)$ \\
\hline \multirow[t]{2}{*}{$\sigma_{12}$} & \multicolumn{2}{|c|}{$-0.011^{*}$} & \multicolumn{2}{|c|}{$-0.008^{*}$} & NA & NA \\
\hline & \multicolumn{2}{|c|}{$(0.002)$} & \multicolumn{2}{|c|}{$(0.002)$} & NA & NA \\
\hline \multirow[t]{2}{*}{$\sigma_{22}$} & \multicolumn{2}{|c|}{$0.026^{*}$} & \multicolumn{2}{|c|}{$0.025^{*}$} & NA & NA \\
\hline & \multicolumn{2}{|c|}{$(0.003)$} & \multicolumn{2}{|c|}{$(0.003)$} & NA & NA \\
\hline No. of Obs. & \multicolumn{2}{|c|}{372} & \multicolumn{2}{|c|}{372} & 372 & 372 \\
\hline
\end{tabular}


$[\mathrm{hp}]$

Table 2

Definition and Summary Statistics of Marketing Variables of the Authentic Firms.

\begin{tabular}{llcc}
\hline & & & \\
\hline Variable & Definition & Mean & SD \\
\hline Cost & Unit Product Cost of High-end Product (US \$) & 33.53 & 19.14 \\
Mkshare & Market Share (\%) & 2.9 & 3.7 \\
BrandNo & The Number of Sub-brands & 1.45 & 0.85 \\
Export & Percentage of Sale Values for Export (\%) & 18.1 & 12.5 \\
PatCost & Patent Application Costs (US \$) & 2453.6 & 1560.1 \\
Ads & Annual Advertisement Expenditure (US \$) & $1,497,700$ & $2,724,200$ \\
Employ & The Number of Employees & 813.7 & 482.6 \\
AW & Total Annual Wages (US \$) & 482.8 & 272.2 \\
\hline
\end{tabular}


Table 3: Estimation Results Under RC-SEM and Different Reduced Models When Z Contains Explanatory Variables.

Note: The table lists the posterior means and standard deviations of model parameters. $\log (d f p h)$ : the logarithm of deflated authentic high-end prices in Chinese Yuan, using the Consumer Price index published in the World Bank World Development Indicators (WDI) (Year 1995 was set as the base year in the database, i.e. CPI=100 in 1995). $f k s h$ : the quantity of counterfeit products in the market faced by the corresponding authentic firm, divided by the sale quantity of this authentic firm. $f k s h_{S T}$ and $f k s h_{L T}$ refer to its short-term and long-term effects, respectively. RC-SEM: the simultaneous equation model with random changepoints. SEM: the standard simultaneous equation model assuming a common response time of two years. RC: random-changepoint model. OLS: the standard OLS model assuming a common response time of two years. "*" indicates that $95 \%$ credible interval excludes zero.

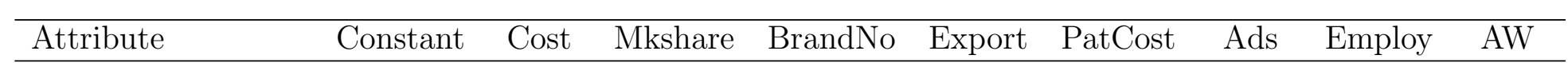

\section{(1) RC-SEM}

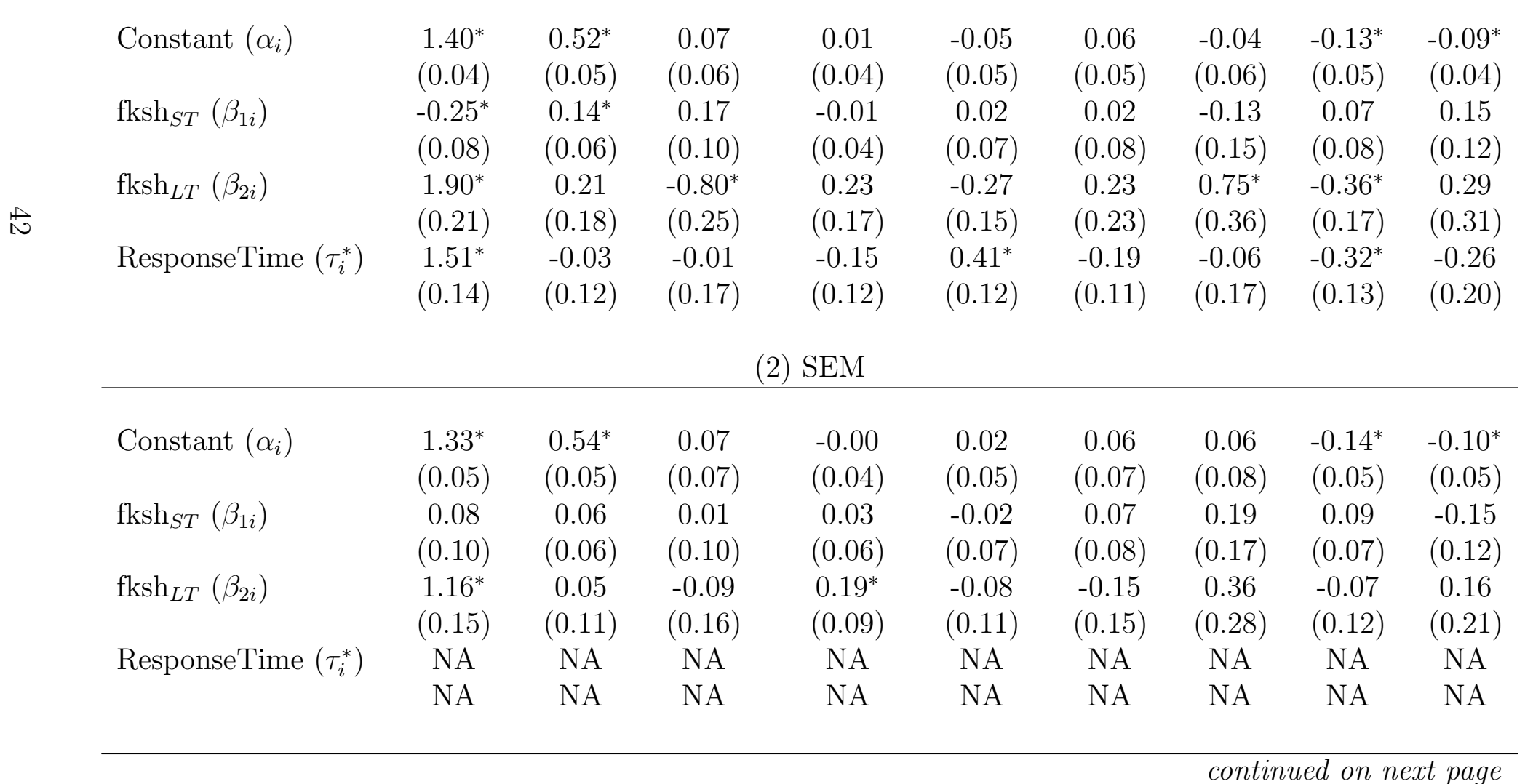


Table 3: continued

\begin{tabular}{|c|c|c|c|c|c|c|c|c|c|}
\hline Attribute & Constant & Cost & Mkshare & BrandNo & Export & PatCost & Ads & Employ & AW \\
\hline \multicolumn{10}{|c|}{ (3) $\mathrm{RC}$} \\
\hline Constant $\left(\alpha_{i}\right)$ & $\begin{array}{l}1.45^{*} \\
(0.05)\end{array}$ & $\begin{array}{l}0.54^{*} \\
(0.05)\end{array}$ & $\begin{array}{c}0.11 \\
(0.07)\end{array}$ & $\begin{array}{c}0.02 \\
(0.04)\end{array}$ & -0.05 & $\begin{array}{c}0.03 \\
(0.07)\end{array}$ & $\begin{array}{l}-0.06 \\
(0.07)\end{array}$ & $-0.13^{*}$ & $\begin{array}{c}-0.11^{*} \\
(0.05)\end{array}$ \\
\hline \multirow{2}{*}{$\mathrm{fksh}_{S T}\left(\beta_{1 i}\right)$} & $-0.61^{*}$ & 0.10 & $0.21^{*}$ & -0.04 & -0.02 & 0.06 & -0.18 & 0.13 & 0.16 \\
\hline & $(0.11)$ & $(0.07)$ & $(0.10)$ & $(0.07)$ & $(0.07)$ & $(0.09)$ & $(0.14)$ & $(0.08)$ & $(0.11)$ \\
\hline \multirow[t]{2}{*}{$\mathrm{fksh}_{L T}\left(\beta_{2 i}\right)$} & $1.24^{*}$ & 0.12 & $-0.48^{*}$ & 0.18 & -0.16 & 0.23 & $0.76^{*}$ & -0.23 & 0.19 \\
\hline & $(0.20)$ & $(0.15)$ & $(0.21)$ & $(0.14)$ & $(0.15)$ & $(0.19)$ & $(0.27)$ & $(0.17)$ & $(0.27)$ \\
\hline \multirow[t]{2}{*}{ ResponseTime $\left(\tau_{i}^{*}\right)$} & $1.52^{*}$ & -0.12 & 0.03 & -0.15 & $0.25^{*}$ & -0.15 & -0.08 & $-0.25^{*}$ & -0.15 \\
\hline & $(0.10)$ & $(0.10)$ & $(0.12)$ & $(0.09)$ & $(0.09)$ & $(0.13)$ & $(0.15)$ & $(0.11)$ & $(0.18)$ \\
\hline \multicolumn{10}{|c|}{ (4) OLS } \\
\hline \multirow[t]{2}{*}{ Constant $\left(\alpha_{i}\right)$} & $1.41^{*}$ & $0.54^{*}$ & 0.08 & 0.01 & 0.02 & 0.05 & -0.02 & $-0.14^{*}$ & $-0.11^{*}$ \\
\hline & $(0.05)$ & $(0.05)$ & $(0.07)$ & $(0.04)$ & $(0.05)$ & $(0.07)$ & $(0.08)$ & $(0.05)$ & $(0.05)$ \\
\hline \multirow[t]{2}{*}{$\mathrm{fksh}_{S T}\left(\beta_{1 i}\right)$} & $-0.21^{*}$ & 0.01 & 0.02 & 0.04 & -0.13 & 0.04 & 0.16 & 0.06 & -0.12 \\
\hline & $(0.09)$ & $(0.07)$ & $(0.10)$ & $(0.06)$ & $(0.07)$ & $(0.08)$ & $(0.17)$ & $(0.07)$ & $(0.12)$ \\
\hline \multirow[t]{2}{*}{$\mathrm{fksh}_{L T}\left(\beta_{2 i}\right)$} & $0.96^{*}$ & 0.04 & -0.15 & $0.21^{*}$ & 0.02 & 0.04 & 0.44 & -0.07 & 0.15 \\
\hline & $(0.15)$ & $(0.11)$ & $(0.16)$ & $(0.09)$ & $(0.11)$ & $(0.15)$ & $(0.31)$ & $(0.13)$ & $(0.20)$ \\
\hline \multirow[t]{2}{*}{ ResponseTime $\left(\tau_{i}^{*}\right)$} & $\mathrm{NA}$ & $\mathrm{NA}$ & $\mathrm{NA}$ & $\mathrm{NA}$ & $\mathrm{NA}$ & $\mathrm{NA}$ & $\mathrm{NA}$ & $\mathrm{NA}$ & $\mathrm{NA}$ \\
\hline & NA & NA & NA & NA & NA & NA & NA & NA & NA \\
\hline
\end{tabular}


Table 4: A simulation study on the comparison of performance of four models on estimating the dynamic entry effects.

RC-SEM: the simultaneous equation model with random changepoints. SEM: the standard simultaneous equation model assuming a common response time of two years. RC: random-changepoint model. OLS: the standard OLS model assuming a common response time of two years. "Bias" and "SD" in the table are the bias and standard deviation of the estimates (posterior means), respectively, over all the replicates. "RMSE" denotes the root mean squared error. "CR" denotes the coverage rate.

\begin{tabular}{|c|c|c|c|c|c|c|c|c|c|c|c|c|c|c|c|c|}
\hline \multirow[t]{2}{*}{$\rho$} & \multicolumn{4}{|c|}{ RC-SEM } & \multicolumn{4}{|c|}{ SEM } & \multicolumn{4}{|c|}{$\mathrm{RC}$} & \multicolumn{4}{|c|}{ OLS } \\
\hline & Bias & SD & RMSE & CR & Bias & SD & RMSE & CR & Bias & $\mathrm{SD}$ & RMSE & $\mathrm{CR}$ & Bias & $\mathrm{SD}$ & RMSE & $\mathrm{CR}$ \\
\hline \multicolumn{17}{|c|}{ Short-term effect (True value $=-0.4$ ) } \\
\hline 0 & -0.01 & 0.12 & 0.12 & $92 \%$ & 0.16 & 0.14 & 0.21 & $66 \%$ & -0.01 & 0.10 & 0.10 & $92 \%$ & 0.16 & 0.13 & 0.20 & $72 \%$ \\
\hline 0.2 & 0.04 & 0.10 & 0.11 & $88 \%$ & 0.22 & 0.13 & 0.25 & $52 \%$ & 0.11 & 0.09 & 0.14 & $76 \%$ & 0.27 & 0.12 & 0.30 & $32 \%$ \\
\hline 0.5 & 0.03 & 0.10 & 0.11 & $92 \%$ & 0.25 & 0.15 & 0.29 & $46 \%$ & 0.20 & 0.10 & 0.23 & $34 \%$ & 0.38 & 0.16 & 0.41 & $18 \%$ \\
\hline 0.8 & 0.01 & 0.09 & 0.09 & $90 \%$ & 0.25 & 0.14 & 0.27 & $42 \%$ & 0.30 & 0.13 & 0.33 & $18 \%$ & 0.45 & 0.16 & 0.48 & $8 \%$ \\
\hline-0.2 & 0.01 & 0.10 & 0.10 & $98 \%$ & 0.18 & 0.14 & 0.22 & $62 \%$ & -0.05 & 0.09 & 0.11 & $88 \%$ & 0.12 & 0.13 & 0.18 & $78 \%$ \\
\hline-0.5 & 0.02 & 0.10 & 0.10 & $92 \%$ & 0.15 & 0.14 & 0.20 & $70 \%$ & -0.15 & 0.10 & 0.17 & $60 \%$ & 0.03 & 0.14 & 0.15 & $84 \%$ \\
\hline-0.8 & 0.00 & 0.09 & 0.09 & $94 \%$ & 0.10 & 0.12 & 0.15 & $74 \%$ & -0.26 & 0.09 & 0.27 & $16 \%$ & -0.10 & 0.11 & 0.15 & $84 \%$ \\
\hline \multicolumn{17}{|c|}{ Long-term effect $($ True Value $=1.0$ ) } \\
\hline 0 & -0.01 & 0.08 & 0.08 & $94 \%$ & -0.16 & 0.10 & 0.18 & $54 \%$ & -0.01 & 0.06 & 0.07 & $94 \%$ & -0.17 & 0.09 & 0.18 & $46 \%$ \\
\hline 0.2 & 0.00 & 0.09 & 0.09 & $92 \%$ & -0.13 & 0.10 & 0.16 & $66 \%$ & 0.07 & 0.09 & 0.12 & $74 \%$ & -0.07 & 0.10 & 0.12 & $80 \%$ \\
\hline 0.5 & 0.00 & 0.08 & 0.08 & $98 \%$ & -0.11 & 0.09 & 0.14 & $80 \%$ & 0.18 & 0.08 & 0.20 & $24 \%$ & 0.03 & 0.10 & 0.10 & $90 \%$ \\
\hline 0.8 & -0.01 & 0.09 & 0.09 & $86 \%$ & -0.09 & 0.08 & 0.12 & $80 \%$ & 0.30 & 0.10 & 0.31 & $4 \%$ & 0.12 & 0.10 & 0.17 & $60 \%$ \\
\hline-0.2 & -0.01 & 0.09 & 0.09 & $92 \%$ & -0.18 & 0.11 & 0.21 & $50 \%$ & -0.09 & 0.07 & 0.12 & $80 \%$ & -0.25 & 0.10 & 0.27 & $16 \%$ \\
\hline-0.5 & 0.01 & 0.09 & 0.09 & $88 \%$ & -0.19 & 0.10 & 0.21 & $46 \%$ & -0.17 & 0.08 & 0.19 & $36 \%$ & -0.33 & 0.10 & 0.34 & $6 \%$ \\
\hline-0.8 & -0.01 & 0.06 & 0.06 & $98 \%$ & -0.24 & 0.08 & 0.25 & $20 \%$ & -0.31 & 0.08 & 0.31 & $2 \%$ & -0.45 & 0.07 & 0.45 & $0 \%$ \\
\hline
\end{tabular}




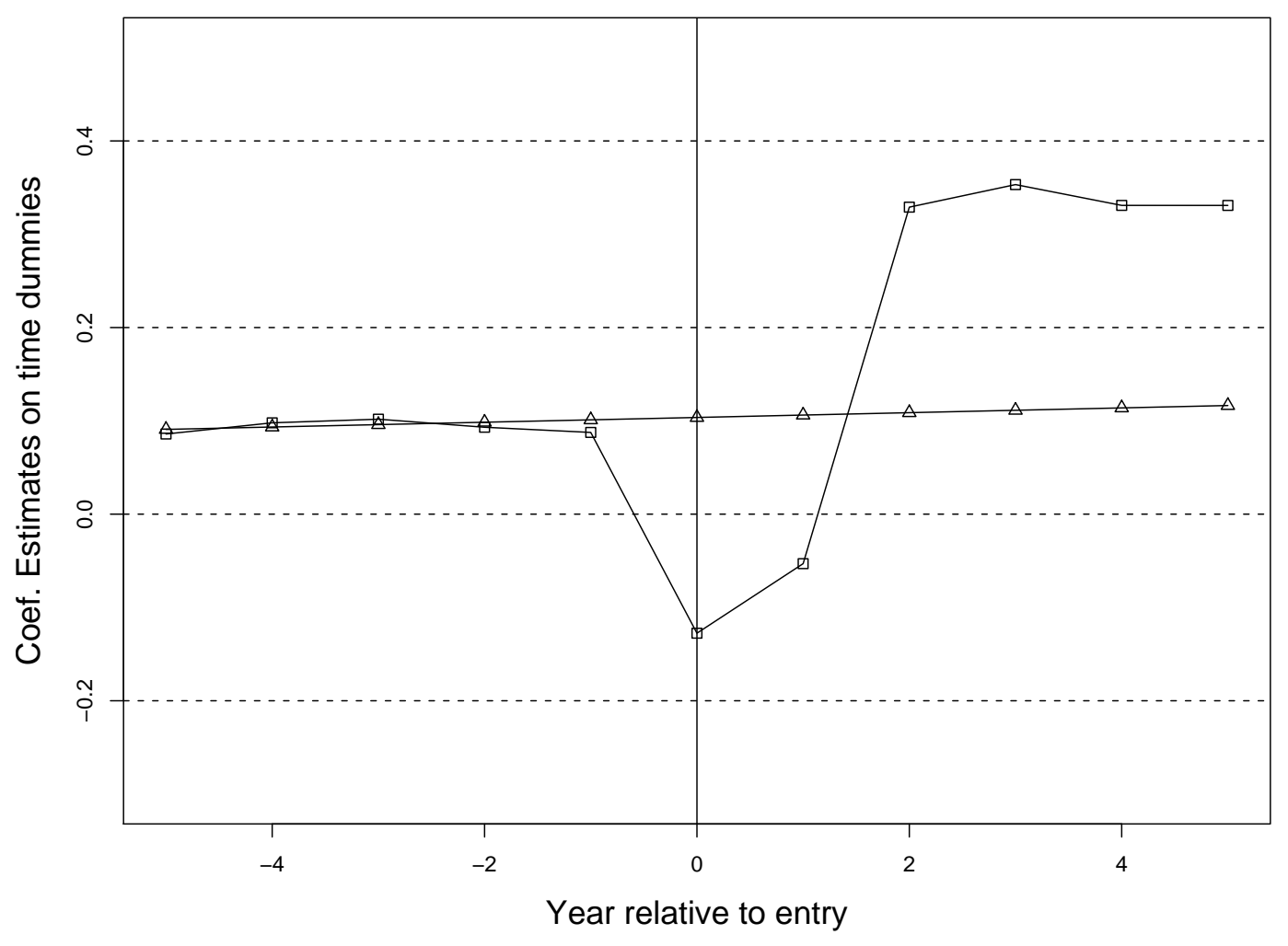

Figure 1. Average log deflated authentic price versus the years relative to the counterfeit entries. $-\square-$ : the regression coefficient estimates on a set of time dummies denoting the number of years relative to the counterfeit entries with the log deflated price for high-end product as the response variable. $-\triangle-$ : prediction based on the pre-entry price trend. 


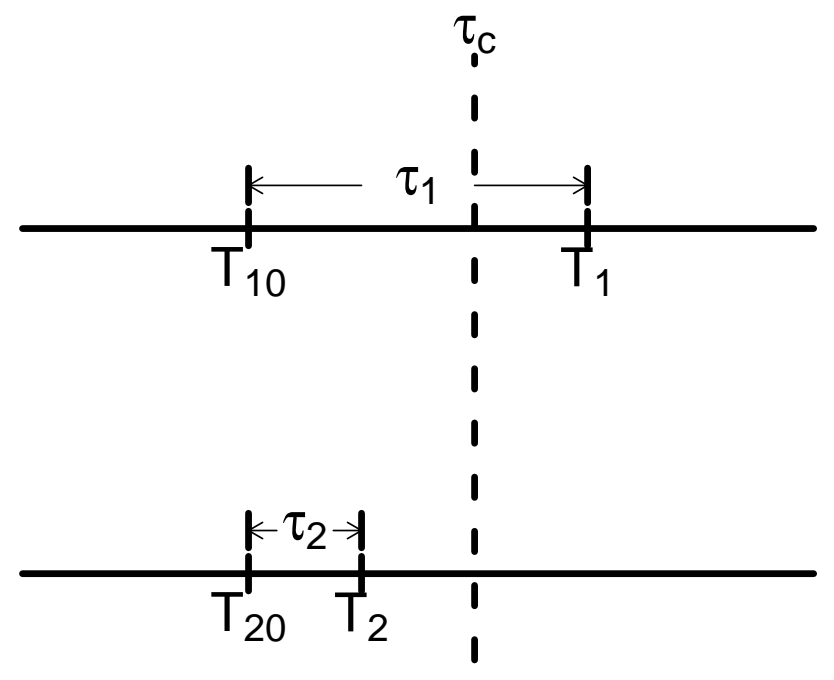

Figure 2. A graph illustrating that ignoring heterogeneity of response times can lead to misleading estimation. The graph shows two hypothetical firms that experience a market change (e.g. counterfeit entry) at time $T_{10}$ and $T_{20}$, respectively. Their latent changepoints in marketing response outcome are $T_{1}$ and $T_{2}$ and the corresponding response time are $\tau_{1}$ and $\tau_{2}$. Pre-specifying a common $\tau_{c}$ ignores this heterogeneity and can lead to incorrect analysis of the dynamic effects of the market change on the response outcome. In contrast, the changepoint model allows for firm-specific changepoint and therefore is well suited for studying heterogeneity of response times across study units. 
$[\mathrm{hP}]$

(a) Prior Distribution of $\tau_{i}^{*}$

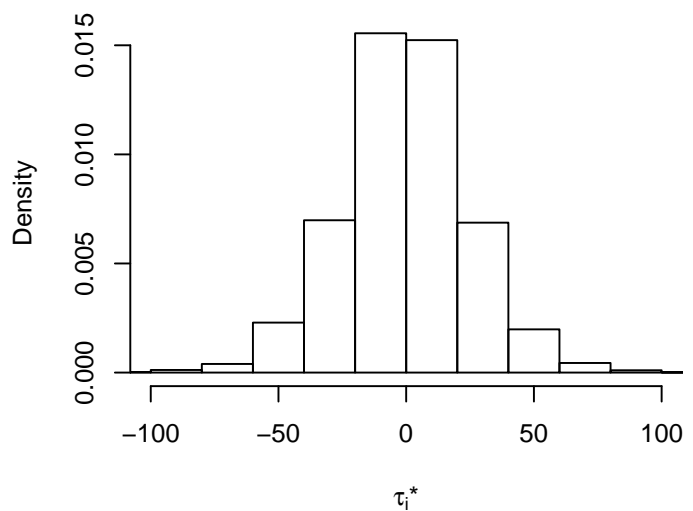

(b) Posterior Distribution of $\tau_{i}^{*}$

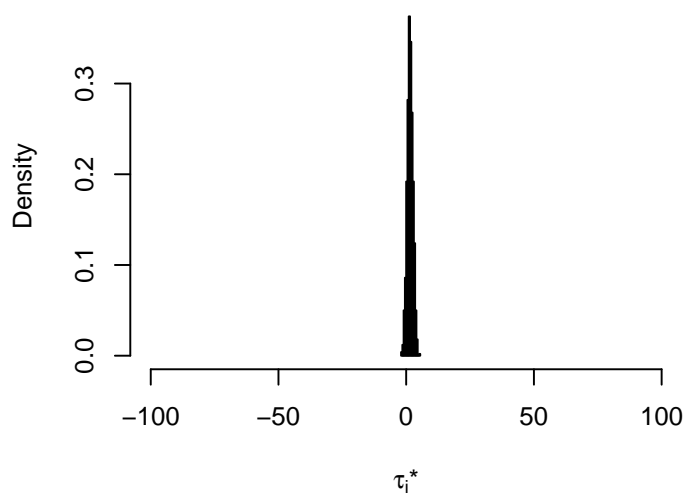

(c) Prior Distribution of $\rho$

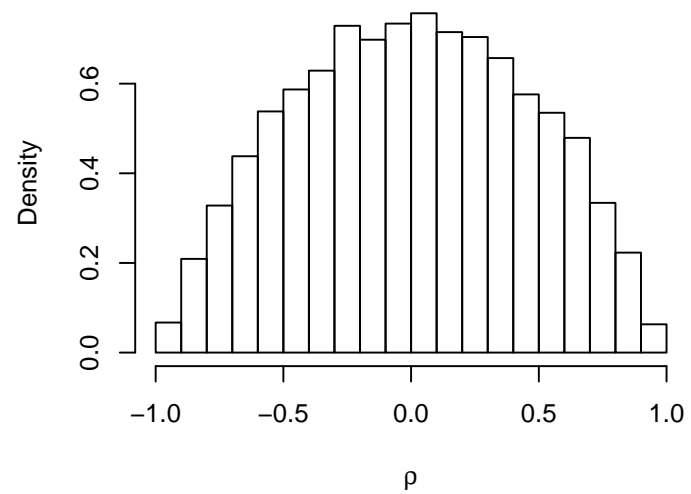

(d) Posterior Distribution of $\rho$

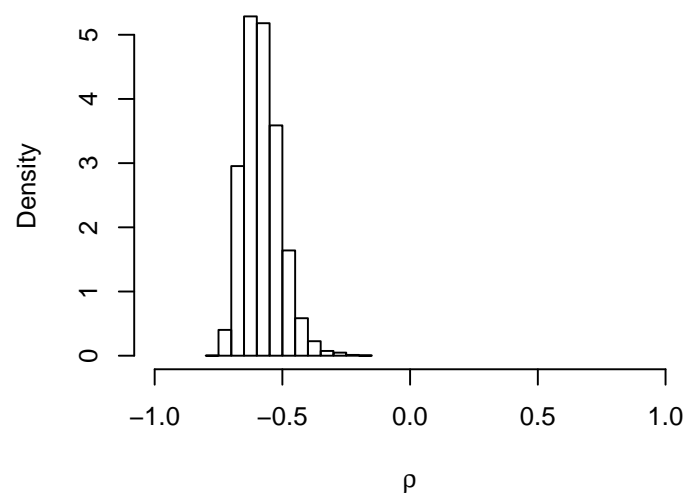

Figure 3. Comparison of Prior and Posterior Distributions of $\tau_{i}^{*}$ and $\rho$. 